\title{
Front Matter: Volume 8785
}

, "Front Matter: Volume 8785," Proc. SPIE 8785, 8th Iberoamerican Optics Meeting and 11th Latin American Meeting on Optics, Lasers, and Applications, 878501 (18 November 2013); doi: 10.1117/12.2050511

SDIE Event: 8th Ibero American Optics Meeting/11th Latin American Meeting on SPIE. Optics, Lasers, and Applications, 2013, Porto, Portugal 


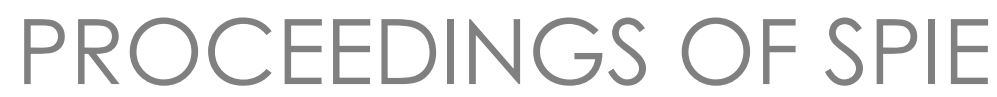

\title{
8th Iberoamerican Optics Meeting and 11 th Latin American Meeting on Optics, Lasers, and Applications
}

\author{
Manuel Filipe P. C. Martins Costa \\ Editor
}

22-26 July 2013

Porto, Portugal

\begin{abstract}
Sponsored by Consortium

Technical Cosponsor and Publisher

SPIE
\end{abstract}

International Commission for Optics - The Optical Society • European Optical Society • Red Iberoamericana de Óptica • Innova Scientific - Álava Ingenieros - Caixa Geral de Depósitos • Messe München GmbH (Germany) - Universidade do Porto (Portugal) - Centro de Láseres Pulsados (Spain) - LASERLAB-EUROPE - Fundação para a Ciência e a Tecnologia (Portugal) • Faculdade de Ciências da Universidade do Porto (Portugal) - FiberSensing, Sistemas Avançados de Monitorização, S.A. (Portugal) - Pl miCos GmbH (Germany) - MT Brandão (Portugal) • Universidade do Minho (Portugal) • Zurich Instruments AG (Switzerland) - Sociedade Portuguesa de Física (Portugal) • APLO (Portugal) • Hands-On Science (Portugal) • La Sociedad Española de Óptica (Spain) - La Academia Mexicana de Óptica, A. C. (Mexico) - Red Colombiana de Óptica (Colombia) - Comité Venezolano De Optica (Venezuela) - La Société Tunisienne d'Optique (Tunisia) - African Laser Atomic Molecular \& Optical Sciences Network - Optics and Photonics Society of Singapore (Singapore) - Photonics21 - Southern European Cluster of Photonics and Optics - LASER World of PHOTONICS (Germany) - European Photonics Industry

Volume 8785 
The papers included in this volume were part of the technical conference cited on the cover and title page. Papers were selected and subject to review by the editors and conference program committee. Some conference presentations may not be available for publication. The papers published in these proceedings reflect the work and thoughts of the authors and are published herein as submitted. The publisher is not responsible for the validity of the information or for any outcomes resulting from reliance thereon.

Please use the following format to cite material from this book:

Author(s), "Title of Paper," in 8th Iberoamerican Optics Meeting and 11 th Latin American Meeting on Optics, Lasers, and Applications, edited by Manuel Filipe P. C. Martins Costa, Proceedings of SPIE Vol. 8785 (SPIE, Bellingham, WA, 2013) Article CID Number.

ISSN: 0277-786X

ISBN: 9780819496010

Published by

SPIE

P.O. Box 10, Bellingham, Washington 98227-0010 USA

Telephone +1 3606763290 (Pacific Time) · Fax +1 3606471445

SPIE.org

Copyright (C) 2013, Society of Photo-Optical Instrumentation Engineers.

Copying of material in this book for internal or personal use, or for the internal or personal use of specific clients, beyond the fair use provisions granted by the U.S. Copyright Law is authorized by SPIE subject to payment of copying fees. The Transactional Reporting Service base fee for this volume is $\$ 18.00$ per article (or portion thereof), which should be paid directly to the Copyright Clearance Center (CCC), 222 Rosewood Drive, Danvers, MA 01923. Payment may also be made electronically through CCC Online at copyright.com. Other copying for republication, resale, advertising or promotion, or any form of systematic or multiple reproduction of any material in this book is prohibited except with permission in writing from the publisher. The CCC fee code is 0277-786X/13/\$18.00.

Printed in the United States of America.

Publication of record for individual papers is online in the SPIE Digital Library.

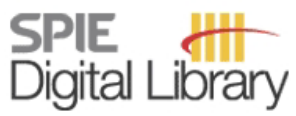

SPIEDigitalLibrary.org

Paper Numbering: Proceedings of SPIE follow an e-First publication model, with papers published first online and then in print and on CD-ROM. Papers are published as they are submitted and meet publication criteria. A unique, consistent, permanent citation identifier (CID) number is assigned to each article at the time of the first publication. Utilization of CIDs allows articles to be fully citable as soon as they are published online, and connects the same identifier to all online, print, and electronic versions of the publication. SPIE uses a six-digit CID article numbering system in which:

- The first four digits correspond to the SPIE volume number.

- The last two digits indicate publication order within the volume using a Base 36 numbering

system employing both numerals and letters. These two-number sets start with 00, 01, 02, 03, 04, $05,06,07,08,09,0 A, 0 B \ldots 0 Z$, followed by 10-1Z, 20-2Z, etc.

The CID Number appears on each page of the manuscript. The complete citation is used on the first page, and an abbreviated version on subsequent pages. Numbers in the index correspond to the last two digits of the six-digit CID Number. 


\section{Contents}

xxxi Conference Committees

xxxvii Introduction

\section{Part One}

\section{PLENARY SESSION I}

878503 Some considerations on the integration methods for Hartmann and Hartmann-Shack patterns (Plenary Paper) [8785-1 18]

G. Hernández-Gómez, D. Malacara-Doblado, Z. Malacara-Hernández, Ctr. de Investigaciones en Óptica, A.C. (Mexico); R. Díaz-Uribe, Univ. Nacional Autónoma de México (Mexico); D. Malacara Hernández, Ctr. de Investigaciones en Óptica, A.C. (Mexico)

\section{SESSION I}

878509 Spatial measurement of spurious forces with optical tweezers [8785-538]

I. Bordeu, Univ. de Concepción (Chile); G. Volpe, Bilkent Univ. (Turkey); J. P. Staforelli, Univ. de Concepción (Chile)

$8785 \mathrm{OA}$ Effective medium theory of subwavelength arrays of metallic nanowires: a numerical approach based on modal propagation method [8785-240]

A. O. Silva, INESC Porto (Portugal) and Univ. Federal do Pará (Brazil); I. T. Leite, INESC Porto (Portugal) and Univ. do Porto (Portugal); J. M. Teixeira, J. P. Araujo, Univ. do Porto (Portugal); J. C. W. A. Costa, Univ. Federal do Pará (Brazil); M. T. R. Giraldi, Instituto Militar de Engenharia (Brazil); P. A. S. Jorge, INESC Porto (Portugal); A. Guerreiro, INESC Porto (Portugal) and Univ. do Porto (Portugal)

\section{SESSION II}

8785 OG End-side-pumped Nd:YAG solar laser with four Fresnel lenses [8785-260]

B. Galinhas, D. Liang, Univ. Nova de Lisboa (Portugal)

\section{SESSION III}

8785 0J Space optical navigation techniques: an overview (Invited Paper) [8785-247]

J. M. Rebordão, Univ. de Lisboa (Portugal) 
$8785 \mathrm{OL}$ Optical design of a Coudé-Train for a stable and efficient simultaneous feeding of the ESPRESSO spectrograph from the four VLT telescopes [8785-287]

A. Cabral, J. Coelho, M. Abreu, Univ. de Lisboa (Portugal); M. Monteiro, Univ. do Porto (Portugal); R. Gomes, P. Santos, A. Oliveira, Univ. de Lisboa (Portugal); G. Ávila,

B.-A. Delabre, European Southern Observatory (Germany); D. Mégevand, Observatoire de I'Univ. de Genève (Switzerland); F. Zerbi, INAF - Osservatorio Astronomico di Brera (Italy); P. Di Marcantonio, INAF - Osservatorio Astronomico di Trieste (Italy); C. Lovis, Observatoire de l'Univ. de Genève (Switzerland); N. C. Santos, Univ. do Porto (Portugal)

$8785 \mathrm{OM}$ Installation of a variable-angle spectrometer system for monitoring diffuse and global solar radiation [8785-152]

O. Ormachea, A. Abrahamse, N. Tolavi, F. Romero, O. Urquidi, Univ. Privada Boliviana (Bolivia); J. M. Pearce, Michigan Technological Univ. (United States); R. Andrews, Queen's Univ. (Canada)

\section{SESSION IV}

8785 OS Observation of Zeeman shift in the rubidium $D_{2}$ line using an optical nanofiber in vapor [8785-500]

A. Watkins, Univ. College Cork (Ireland); V. B. Tiwari, Ctr. for Advanced Technology (India); J. M. Ward, K. Deasy, Okinawa Institute of Science and Technology (Japan);

S. Nic Chormaic, Univ. College Cork (Ireland), Okinawa Institute of Science and Technology (Japan), and Univ. of KwaZulu-Natal (South Africa)

\section{SESSION $V$}

8785 ОT Generation of ultrabroadband energetic laser pulses by noncollinear optical parametric chirped pulse amplification (Invited Paper) [8785-648]

G. Figueira, Univ. Técnica de Lisboa (Portugal); T. Imran, Univ. Técnica de Lisboa (Portugal) and King Saud Univ. (Saudi Arabia); C. P. João, H. Pires, L. Cardoso, Univ. Técnica de Lisboa (Portugal)

8785 OW Core-doped Nd:YAG disk solar laser uniformly pumped by six Fresnel lenses [8785-682]

D. Garcia, D. Liang, J. Almeida, Univ. Nova de Lisboa (Portugal)

\section{SESSION VI}

8785 OY Different applications of liquid crystal panels (Invited Paper) [8785-640]

A. Lizana, A. Peinado, C. Ramírez, Univ. Autònoma de Barcelona (Spain); J. L. Martínez, Univ. Autònoma de Barcelona (Spain) and Univ. Miguel Hernández de Elche (Spain); A. Hussain, M. Sohail, Univ. Autònoma de Barcelona (Spain) and Pakistan Institute of Engineering and Applied Sciences (Pakistan); C. lemmi, Univ. de Buenos Aires (Argentina); A. Márquez, Univ. de Alicante (Spain); I. Moreno, Univ. Miguel Hernández de Elche (Spain); M. J. Yzuel, J. Campos, Univ. Autònoma de Barcelona (Spain) 
878511 Detection of refractive index changes in chemical reactions of fluids within micro channels using digital holographic microscopy [8785-475]

A. Nasehi, S. Moradi Mehr, Univ. of Zanjan (Iran, Islamic Republic of); A.-R. Moradi, Univ. of Zanjan (Iran, Islamic Republic of) and Institute for Advanced Studies in Basic Science (Iran, Islamic Republic of)

\section{SESSION VII}

878517 Optics in gait analysis and anthropometry (Invited Paper) [8785-472]

A. A. Silva Moreno, CIATEC, A.C. (Mexico)

878518 Side-pumping Nd:YAG solar laser by six Fresnel lenses [8785-254]

G. Tomás, D. Liang, J. Almeida, Univ. Nova de Lisboa (Portugal)

8785 IB The validation of the Z-Scan technique for the determination of plasma glucose [8785-234]

S. I. Alves, Univ. Federal de São Paulo (Brazil); E. A. O. Silva, Univ. Federal de São Paulo

(Brazil) and Fundação do $A B C$ (Brazil); S. S. Costa, Fundação do ABC (Brazill);

D. R. N. Sonego, M. L. Hallack, O. L. Coppini, F. Rowies, L. A. Azzalis, V. B. C. Junqueira,

E. C. Pereira, K. C. Rocha, Univ. Federal de São Paulo (Brazil); F. L. A. Fonseca, Univ. Federal de São Paulo (Brazil) and Fundação do ABC (Brazil)

\section{SESSION VIII}

8785 1D Development of a portable low-cost LIBS system (Invited Paper) [8785-157]

O. Ormachea, O. Urquidi, D. Casazola, Univ. Privada Boliviana (Bolivia)

8785 IF INSCAN PRO: a fast ultraviolet spectrometer design approach [8785-361]

B. W. Myer, J. M. Dias, Instituto de Plasmas e Fusão Nuclear (Portugal)

$87851 \mathrm{G}$ Measurement of the roughness surface of the specials papers using the normalized autocorrelation function of the fields of the texture of speckle pattern [8785-542]

A. O. Pino, Univ. Tecnológica de Panama (Panama); J. Pladellorens, Univ. Politècnica de Catalunya (Spain)

\section{SESSION IX}

$8785 \mathrm{lK} \quad$ High resolution optical surface testing using transport of intensity equation [8785-356] P. Soltani, Univ. of Zanjan (Iran, Islamic Republic of); A.-R. Moradi, A. Darudi, Univ. of Zanjan (Iran, Islamic Republic of) and Institute for Advanced Studies in Basic Sciences (Iran, Islamic Republic of); R. Shomali, Univ. of Zanjan (Iran, Islamic Republic of)

$87851 \mathrm{M}$ Degree of polarization as a cross-correlation detector for absolute distance measurement [8785-561]

D. Castro Alves, M. Abreu, A. Cabral, J. M. Rebordão, Univ. of Lisbon (Portugal) 
8785 iN Micro fluid flow visualization of a circular nozzle by using the Schlieren technique [8785-276]

C. Alvarez-Herrera, Univ. Autónoma de Chihuahua (Mexico); D. Moreno-Hernández, Ctr. de Investigaciones en Óptica, A.C. (Mexico); J. G. Murillo-Ramírez, Ctr. de Investigación en Materiales Avanzados, S.C. (Mexico)

POSTER SESSION I

878510 Fluorescence support in optical tweezers [8785-1]

J. G. Animas, M. Arronte, T. Flores, L. Ponce, Ctr. de Investigación en Ciencia Aplicada y Tecnología Avanzada (Mexico)

8785 IP Grating optical filter for pre-adapted angular selective regulation of directional light transmission [8785-10]

R. S. Zakirullin, Orenburg State Univ. (Russian Federation)

$87851 Q \quad$ Image encryption-decryption system based on gyrator transform and jigsaw transform [8785-100]

J. M. Vilardy, Univ. Politècnica de Catalunya (Spain) and Univ. Popular del Cesar

(Colombia); C. O. Torres, L. Mattos, Univ. Popular del Cesar (Colombia)

8785 IR Double image encryption method using the Arnold transform in the fractional Hartley domain [8785-101]

J. M. Vilardy, Univ. Politècnica de Catalunya (Spain) and Univ. Popular del Cesar (Colombia); C. O. Torres, Univ. Popular del Cesar (Colombia); C. J. Jimenez, Univ. de La Guajira (Colombia)

8785 IT Implementation of a semi-automatic robotic system for alignment in the Ronchi test [8785-105]

Y. Maldonado-Pérez, A. Santiago-Alvarado, J. González-García, Univ. Technológica de la Mixteca (Mexico); A. Cordero-Dávila, Benemérita Univ. Autónoma de Puebla (Mexico); V. Rybak, Univ. Technológica de la Mixteca (Mexico)

$87851 \mathrm{U}$ Weighted average of the Gouy phase shift and their relation with the irradiance moments of a beam [8785-107]

R. Martínez-Herrero, Univ. Complutense de Madrid (Spain); A. Manjavacas, Instituto de Química-Física Rocasolano (Spain)

8785 IV Elliptical Bessel-like diffraction pattern produced by circular apertures with different radius [8785-108]

E. Andrés-Zárate, Q. Angulo-Córdova, J. A. Hernández-Nolasco, G. Gutiérrez-Tepach, Univ. Juárez Autónoma de Tabasco (Mexico); C. G. Treviño-Palacios, Instituto Nacional de Astrofísica, Óptica y Electrónica (Mexico)

$87851 \mathrm{X}$ Analysis of strawberry ripening by dynamic speckle measurements [8785-111] C. Mulone, Univ. Tecnológica Nacional (Argentina); N. Budini, Univ. Tecnológica Nacional (Argentina) and Instituto de Física del Litoral (Argentina); F. M. Vincitorio, C. Freyre, Univ. Tecnológica Nacional (Argentina); A. J. López Díaz, A. Ramil Rego, Univ. da Coruña (Spain) 
8785 IY Synchronization of optically coupled resonant tunneling diode oscillators [8785-1 12] B. Romeira, J. M. L. Figueiredo, Univ. do Algarve (Portugal); C. N. Ironside, Univ. of Glasgow (United Kingdom); J. M. Quintana, Instituto de Microelectrónica de Sevilla (Spain)

878512 Analysis and design of a mechanical system to use with the Ronchi and Fizeau tests [8785-113]

A. D. Galán-Martínez, A. Santiago-Alvarado, J. González-García, V. M. Cruz-Martínez, Univ. Technológica de la Mixteca (Mexico); A. Cordero-Dávila, Benemérita Univ. Autónoma de Puebla (Mexico); F. S. Granados-Agustin, Instituto Nacional de Astrofísica, Óptica y Electrónica (Mexico); C. Robledo-Sánchez, Benemérita Univ. Autónoma de Puebla (Mexico)

878523 Modulator for a micro Mach-Zhender spectrometer [8785-12]

A. G. Rojas-Hernández, R. Gómez-Fuentes, A. García-Juárez, D. Berman-Mendoza, A. Vera-Marquina, L. A. Garcia-Delgado, A. L. Leal-Cruz, I. E. Zaldivar-Huerta, Univ. de Sonora (Mexico)

878526 Determining the virtual surface in the thermal evaporation process of magnesium fluoride from a tungsten boat for different deposition rates, to be used in precision optical components [8785-122]

A. Tejada Esteves, G. Gálvez de la Puente, Pontificia Univ. Católica del Perú (Peru)

878527 Effects of rotation in the laser-pulse photoassociation in a thermal gas of atoms [8785-123] E. F. de Lima, Univ. Estadual Paulista (Brazil)

8785 2C Determining the quality of optical fiber splice by fusion by electric arc through spectral image processing [8785-135]

F. Vega, C. Torres, L. Diaz, L. Mattos, Univ. Popular del Cesar (Colombia)

8785 2D Simple method of fabrication tapered fiber [8785-137]

F. Vega, C. Torres, L. Diaz, L. Mattos, Univ. Popular del Cesar (Colombia)

$87852 \mathrm{~F}$ System for measuring the angular response of radiometers [8785-14]

L. A. Berni, W. A. Vilela, A. F. Beloto, F. O. de Sena, Instituto Nacional de Pesquisas Espaciais (Brazil)

$87852 \mathrm{G}$ Light pollution and solid-state lighting: reducing the carbon dioxide footprint is not enough [8785-140]

S. Bará, Univ. de Santiago de Compostela (Spain)

$87852 \mathrm{~J}$ Experimental method to characterize a liquid-crystal variable retarder and its application in a Stokes polarimeter. [8785-149]

J. M. López-Téllez, N. C. Bruce, Univ. Nacional Autónoma de México (Mexico)

$87852 \mathrm{~L}$ Detection of wavelengths in the visible range using fiber optic sensors [8785-156]

L. Díaz, Y. Morales, L. Mattos, C. O. Torres, Univ. Popular del Cesar (Colombia) 
$87852 \mathrm{M}$ Holographic interferometry as a method to detect welding failures on ribbed iron structures [8785-160]

F. M. Vincitorio, Univ. Tecnológica Nacional (Argentina); N. Budini, Univ. Tecnológica Nacional (Argentina) and Instituto de Física del Litoral (Argentina); C. Mulone, C. Freyre, M. Spector, Univ. Tecnológica Nacional (Argentina); A. J. López Díaz, A. Ramil Rego, A. Yañez Casal, Univ. da Coruña (Spain)

$87852 \mathrm{~N} \quad$ Holographic recording in photorefractive $\mathrm{Bi}_{2} \mathrm{TeO}_{5}$ crystals at high intensity [8785-164] I. de Oliveira, Univ. Estadual de Campinas (Brazil); J. F. Carvalho, Z. V. Fabris, Univ. Federal de Goías (Brazil); J. Frejlich, Univ. Estadual de Campinas (Brazil)

878520 Homogeneous polarized light by non-quadrature amplitude modulation [8785-168] G.-P. Lemus-Alonso, C. Meneses-Fabian, R. Kantun-Montiel, U. Rivera-Ortega, F.-A. Lara-Cortes, R. Juarez-Salazar, C. Robledo-Sánchez, Benemérita Univ. Autónoma de Puebla (Mexico)

$87852 \mathrm{P} \quad$ Polarized light by quadrature amplitude modulation [8785-169] R. Kantun-Montiel, C. Meneses-Fabian, U. Rivera-Ortega, G.-P. Lemus-Alonso, E. Barojas-Gutierrez, R. Pastrana-Sanchez, Benemérita Univ. Autónoma de Puebla (Mexico)

$87852 \mathrm{R} \quad$ Packaging and testing of fiber Bragg gratings for use as strain sensor in rock specimens [8785-171]

A. Castro-Caicedo, P. Torres, Univ. Nacional de Colombia (Colombia); R. Lain, Univ. Politécnica de Madrid (Spain)

878525 Tridimensional ablation region in femtosecond laser micromachining with diffractive lenses [8785-175]

S. Torres-Peiró, J. González-Ausejo, O. Mendoza-Yero, G. Mínguez-Vega, J. Lancis, Univ. Jaume I (Spain)

$87852 \mathrm{U}$ On-axis Dammann lenses and femtosecond pulses: triggering non-linear effects [8785-177]

J. Pérez-Vizcaíno, O. Mendoza-Yero, Univ. Jaume I (Spain); R. Borrego-Varillas, Univ. de Salamanca (Spain); G. Mínguez-Vega, Univ. Jaume I (Spain); J. R. Vázquez de Aldana, Univ. de Salamanca (Spain); P. Andrés, Univ. de València (Spain); J. Láncis, Univ. Jaume I (Spain)

8785 2W Propagation of Gaussian beams through active layers [8785-180]

V. Vázquez, J. C. Fernández, M. T. Garea, Univ. de Buenos Aires (Argentina); C. L. Matteo, L. I. Perez, Univ. de Buenos Aires (Argentina) and Consejo Nacional de Investigaciones Científicas y Técnicas (Argentina); P. A. Sorichetti, Univ. de Buenos Aires (Argentina)

$87852 Y \quad$ Accuracy of the optical testing of a fast aspheric concave surface through null-screens [8785-182]

M. Campos-García, Univ. Nacional Autónoma de México (Mexico); F. Granados-Agustín, E. Percino-Zacarías, Instituto Nacional de Astrofísica, Óptica y Electrónica (Mexico); A. Estrada-Molina, Univ. Nacional Autónoma de México (Mexico); V. I. Moreno-Oliva, Univ. del Istmo (Mexico); A. Santiago-Alvarado, Univ. Technológica de la Mixteca (Mexico); M. Avendaño-Alejo, Univ. Nacional Autónoma de México (Mexico) 
878532 Double-scatter Mueller matrices for vector electromagnetic scattering from single grooves and lines on rough surfaces [8785-186]

N. C. Bruce, Univ. Nacional Autónoma de México (Mexico)

878534 Tracking of wet foam ageing by means of dynamic laser speckle and computer optical mouse [8785-188]

J. Guerrero, A. Plata Planidina, E. Mejía-Ospino, R. Cabanzo, Univ. Industrial de Santander (Colombia)

878535 Control of a piezoelectric device and image system acquisition for introducing phase steps in interferential microscope [8785-189]

J. Choque A., M. Asmad, V. Sarmiento, G. Baldwin, Pontificia Univ. Católica del Perú (Peru)

878538 Metallic coating techniques for fiber Bragg grating sensors [8785-197]

T. Grandal, E. Piñeiro, A. Asensio, F. Rodriguez, Asociación de Investigación Metalúrgica del Noroeste (Spain)

878539 Analysis of temporal stability of autostereoscopic 3D displays [8785-198] M. Rubiño, C. Salas, A. M. Pozo, J. J. Castro, F. Pérez-Ocón, Univ. de Granada (Spain)

8785 3B Spectroradiometric characterization of autostereoscopic 3D displays [8785-201] M. Rubiño, C. Salas, A. M. Pozo, J. J. Castro, F. Pérez-Ocón, Univ. de Granada (Spain)

$87853 \mathrm{D}$ Operating principle of a high resolution ultrasonic ranging system based in a phase processing [8785-204]

L. X. Chaparro, C. R. Contreras, J. E. Meneses, Univ. Industrial de Santander (Colombia)

$87853 \mathrm{H} \quad$ Fourier transform methods applied to an optical heterodyne profilometer [8785-218]

A. Beltrán-González, G. García-Torales, Univ. de Guadalajara (Mexico);

G. Martínez-Ponce, Ctr. de Investigaciones en Óptica, A.C. (Mexico)

$87853 \mathrm{~J}$ Joint transform correlator-based encryption system using the Fresnel transform and nonlinear filtering [8785-22]

J. M. Vilardy, M. S. Millán, E. Pérez-Cabré, Univ. Politècnica de Catalunya (Spain)

878530 Static and dynamic amplitude modulation of light in a twisted nematic liquid crystal display [8785-23]

J. M. Vilardy, M. S. Millán, E. Pérez-Cabré, Univ. Politècnica de Catalunya (Spain)

$87853 Q \quad$ Nonlinear optical study of p-bromo-benzylidene aniline-substituted [8785-233]

A. K. C. A. Reis, D. R. Paiva, M. M. Reginato, S. I. Alves, Univ. Federal de São Paulo (Brazil)

$878535 \quad$ Measurement of the dispersion in special optical fibers [8785-239]

G. Beltrán Pérez, T. J. Cerdá Astorga, I. Armas Rivera, J. I. Vazquez Lozano,

J. Castillo Mixcóatl, S. Muñoz Aguirre, Benemérita Univ. Autónoma de Puebla (Mexico)

$87853 \mathrm{~T}$ Reflectance of interurban-road pavements from radar-based measurements [8785-24]

P. Fernandez, A. Peña-García, Univ. of Granada (Spain); D. Poyatos-Martínez,

D. Escot-Bocanegra, National Institute for Aerospace Technology (Spain);

A. Fernández-Oliveras, Univ. of Granada (Spain) 
8785 3W Two step parallel phase shifting interferometry using a double cyclic shear interferometer [8785-249]

N.-I. Toto-Arellano, Univ. Tecnológica de Tulancingo (Mexico); D.-I. Serrano-García,

Utsunomiya Univ. (Japan) and Ctr. de Investigaciones en Óptica, A.C. (Mexico)

$87853 Z$ Extending temporal coherence in speckle interferometry [8785-256]

J. M. Crespo Contiñas, V. Moreno de las Cuevas, M. Gallas Torreira, M. Calizaya Calizaya, Univ. de Santiago de Compostela (Spain)

878542 Automatic real-time generalized phase-shifting interferometry to process interferograms with spatio-temporal visibility [8785-262]

R. Juarez-Salazar, C. Robledo-Sanchez, C. Meneses-Fabian, G. Rodriguez-Zurita,

F. Guerrero Sanchez, A. Barcelata-Pinzon, Benemérita Univ. Autónoma de Puebla (Mexico)

878543 Straightforward filtering to phase demodulation by a Fourier normalized-fringe approach [8785-264]

R. Juarez-Salazar, C. Robledo-Sanchez, C. Meneses-Fabian, G. Rodriguez-Zurita,

F. Guerrero-Sanchez, Benemérita Univ. Autónoma de Puebla (Mexico);

J. Gonzalez-Garcia, Univ. Technológica de la Mixteca (Mexico)

878545 Laser processing of phenolic wood substitutes [8785-268]

F. Quintero, Univ. de Vigo (Spain); A. Riveiro, Ctr. Univ. de la Defensa (Spain); F. Lusquiños, J. Penide, F. Arias-González, J. del Val, R. Comesaña, M. Boutinguiza, J. Pou, Univ. de Vigo (Spain)

878546 Processing of pure Ti by rapid prototyping based on laser cladding [8785-269]

F. Arias-González, J. del Val, R. Comesaña, F. Lusquiños, F. Quintero, Univ. de Vigo (Spain); A. Riveiro, Ctr. Univ. de la Defensa (Spain); M. Boutinguiza, J. Pou, Univ. de Vigo (Spain)

878547 Analysis of the propagation of flat-topped beam in nonlinear optical fiber [8785-27] D. A. Avila, C. O. Torres, Univ. Popular del Cesar (Colombia)

8785 4B Influence of irradiation parameters in nanosecond Nd:YVO4 laser micro-machining of stainless steel for biomedical applications [8785-280]

M. P. Fiorucci, A. J. López, A. Ramil, Univ. da Coruña (Spain)

8785 4C Color dependence with horizontal-viewing angle and colorimetric characterization of two displays using different backlighting [8785-283]

J. J. Castro, A. M. Pozo, M. Rubiño, Univ. de Granada (Spain)

$87854 \mathrm{D}$ Experimental characterization of a highlly nonlinear fiber [8785-289]

A. Supe, Riga Technical Univ. (Latvia); G. Fernandes, N. Muga, A. Pinto, Instituto de

Telecomunicações (Portugal); M. Ferreira, Univ. de Aveiro (Portugal)

$87854 \mathrm{~F}$ Characterization of the spatial resolution in two-photon spectroscopic techniques used for plasma diagnostics [8785-292]

M. Garcia-Lechuga, Univ. de Valladolid (Spain) and Instituto de Óptica "Daza de Valdés" (Spain); L. M. Fuentes, K. Gruetzmacher, C. Perez, M. I. de la Rosa, Univ. de Valladolid (Spain)

8785 4G White light interferometry applied to wavelength calibration of spectrometers [8785-293]

H. González-Núñez, R. de la Fuente, Univ. de Santiago de Compostela (Spain) 
$87854 \mathrm{H} \quad$ Design of microstructured optical fibers for supercontinuum generation [8785-295]

S. M. Rodrigues, M. M. Facão, S. C. Latas, M. F. S. Ferreira, Univ. de Aveiro (Portugal)

87854 Four-wave-mixing assisted pulse shaping of femtosecond UV pulses [8785-298]

M. Canhota, H. Crespo, Univ. do Porto (Portugal); R. Weigand, Univ. Complutense de Madrid (Spain)

$87854 \mathrm{~J}$ Solvent effects on the optical properties of two-level systems with permanent dipole moments [8785-30]

A. Mastrodomenico, M. A. Izquierdo, J. L. Paz, Univ. Simón Bolívar (Venezuela);

P. Colmenares, Univ. de Los Andes (Venezuela)

$87854 \mathrm{M}$ All-optical phase modulated format conversion for high transmission rates based on fiber nonlinearity [8785-306]

V. C. Duarte, M. V. Drummond, R. N. Nogueira, Instituto de Telecomunicações (Portugal)

$87854 \mathrm{~N}$ Dynamic demagnetization in magnetic thin films using a new pump-probe system in the 6 femtosecond range [8785-308]

C. S. Gonçalves, A. S. Silva, H. Crespo, D. S. Schmool, Univ. do Porto (Portugal)

878540 Configurable multipulsing of a MOPA pulsed fiber laser with applications in materials processing [8785-309]

P. T. Guerreiro, J. M. Sousa, R. Romero, M. Silva, A. Duarte, J. R. Salcedo, Multiwave

Photonics (Portugal)

$87854 \mathrm{P} \quad$ Propagation of the four-wave mixing signal immersed in a thermal bath [8785-31]

M. A. Izquierdo, A. Mastrodomenico, J. L. Paz, Univ. Simón Bolívar (Venezuela)

\section{Part Two}

$87854 R$ Design, performance, and tolerances of an active GRIN laser beam shaper [8785-313] A. I. Gómez-Varela, C. Bao-Varela, M. T. Flores-Arias, Instituto de Ciencia de Materiales de Aragón (Spain) and Univ. de Santiago de Compostela (Spain)

87854 Characterization of the thermal refraction in ionic liquids induced by a train of femtosecond laser pulses [8785-316]

E. López Lago, Univ. de Santiago de Compostela (Spain); J. A. Nóvoa, Univ. de Vigo (Spain); D. Nóvoa, Ctr. de Láseres Pulsados (Spain); H. Michinel, Univ. de Vigo (Spain); O. Cabeza, Univ. da Coruña (Spain); J. R. Rodríguez, L. M. Varela, Univ. de Santiago de Compostela (Spain)

8785 4T Devil's vortex-lens arrays generating 3D optical vortex structures [8785-318]

A. Calabuig, S. Sánchez-Ruiz, L. Martínez-León, E. Tajahuerce, M. Fernández-Alonso, Univ. Jaume I (Spain); W. Furlan, A. Pons-Martí, Univ. de València (Spain)

$87854 \mathrm{X}$ Automation methodology for the development of LPFG using $\mathrm{CO}_{2}$ laser radiation [8785-326]

D. Castro Alves, J. M. P. Coelho, M. Nespereira, F. Monteiro, M. Abreu, J. M. Rebordão, Univ. de Lisboa (Portugal) 
$87854 Z$ Optical fiber tapers produced by near-infrared laser radiation [8785-333]

M. Nespereira, J. M. P. Coelho, F. Monteiro, M. Abreu, J. M. Rebordão, Univ. de Lisboa (Portugal)

878551 Interferometric beating in digital Fourier transform holography [8785-337]

G. N. Oliveira, M. E. de Oliveira, J. Dias, P. A. M. dos Santos, Univ. Federal Fluminense (Brazil)

878556 Photorefractive moiré-like patterns with different variation directions for multi-projection in profilometer applications [8785-346]

G. N. Oliveira, M. E. de Oliveira, J. Dias, P. A. M. dos Santos, Univ. Federal Fluminense (Brazil)

8785 5E Stabilization and calibration of an ECDL system with a Michelson interferometer [8785-360]

I. Outumuro, J. L. Valencia, Lab. Oficial de Metroloxía de Galicia (Spain); J. Diz-Bugarin,

Univ. de Vigo (Spain); I. Estevez-Caride, Lab. Oficial de Metroloxía de Galicia (Spain);

J. Blanco, B. V. Dorrío, Univ. de Vigo (Spain)

878551 Spatial resolution in time domain imaging for different phantom widths using the cumulant expansion solution to the transport equation [8785-367]

E. Ortiz-Rascón, N. C. Bruce, A. A. Rodríguez-Rosales, J. Garduño-Mejía, R. Ortega-Martínez, Univ. Nacional Autónoma de México (Mexico)

$87855 \mathrm{~L} \quad$ Study of factors affecting the appearance of colors under microscopes [8785-261] R. Zakizadeh, Univ. of Eastern Finland (Finland); J. Martinez-Garcia, K. B. Raja, C. Siakidis, Gjøvik Univ. College (Norway)

\section{SESSION X}

878550 Broadband coherent mid-IR supercontinuum generation using highly nonlinear tapered photonic crystal fibers [8785-524]

A. Ben Salem, R. Cherif, M. Zghal, SUP'COM (Tunisia) and GreS'Com Lab. (Tunisia)

\section{SESSION XII}

$87855 X \quad$ Identification of retinal cells in in-vivo high resolution images [8785-505] P. Rangel-Fonseca, Ctr. de Investigaciones en Óptica, A.C. (Mexico); A. Gómez-Vieyra, Univ. Autónoma Metropolitana (Mexico); D. Malacara-Hernández, J. C. Estrada-Rico, G. Henández-Gómez, Ctr. de Investigaciones en Óptica, A.C. (Mexico)

$87855 \mathrm{Y}$ Hartmann and Hartmann-Shack spot identification and centroid evaluation by a new efficient segmentation and thresholding algorithm [8785-510] G. Hernández-Gómez, Z. Malacara-Hernández, D. Malacara-Hernández, Ctr. de Investigaciones en Óptica, A.C. (Mexico); A. Gómez-Vieyra, Univ. Autónoma Metropolitana (Mexico) 
878566 Optical fiber trap: radiation pressure effect on inorganic and organic micro-particles (Invited Paper) [8785-432]

H. H. Cerecedo-Núñez, J. C. Alvarado-Zacarías, P. Padilla-Sosa, Univ. Veracruzana (Mexico); M. A. Basurto-Pensado, Univ. Autónoma del Estado de Morelos (Mexico); M. May-Alarcón, Univ. Autónoma del Carmen (Mexico); R. Ramos-García, Instituto Nacional de Astrofísica, Óptica y Electrónica (Mexico); L. Beltrán-Parrazal, Univ. Veracruzana (Mexico)

878568 Evaluation between methods for the color measurement in holograms by using a CMOSRGB camera and a spectrometer [8785-550]

N. Palacios-Ortega, D. Velásquez Prieto, Univ. EAFIT (Colombia)

\section{SESSION XIV}

87856 A Design of new phase shifting algorithms according to the interferometer sensitivities (Invited Paper) [8785-321]

M. Miranda, J. Diz-Bugarín, B. V. Dorrío, J. Blanco, Univ. de Vigo (Spain)

\section{SESSION XV}

$87856 \mathrm{~J}$ Design and testing under actual condition of performance of an optical voltage measurement transformer for transmission lines electrical of high voltage [8785-210] N. Gomez, H. Salazar, A. Ciro, Instituto Tecnológico Metropolitano (Colombia)

\section{SESSION XVI}

$87856 Q \quad$ Curvature phase factor in digital holographic microscopy [8785-286]

A. Doblas, E. Sánchez-Ortiga, M. Martínez-Corral, G. Saavedra, P. Andres, Univ. de València (Spain); J. Garcia-Sucerquia, Univ. de València (Spain) and Univ. Nacional de Colombia Sede Medellín (Colombia)

8785 6S Coupling for capturing an displaying hologram systems for real-time digital holographic interferometry [8785-63]

R. Porras-Aguilar, W. Zaperty, M. Kujawinska, Warsaw Univ. of Technology (Poland)

$87856 \mathrm{U}$ Morphologic evaluation of thin films by algorithms of optical phase stepping applied to images obtained by interferential microscopy [8785-232]

V. Sarmiento, M. Asmad, J. I. Choque, G. Baldwin, Pontificia Univ. Católica del Perú (Peru)

SESSION XVII

$87856 \mathrm{~W}$ Optimization of distributed bolometers coupled to optical antennas in the infrared (Invited Paper) [8785-652]

A. Cuadrado, M. Silva-Lopez, Univ. Complutense de Madrid (Spain); F. J. González, Univ. Autónoma de San Luis Potosi (Mexico); J. Alda, Univ. Complutense de Madrid (Spain) 
878572 RGB digital lensless holographic microscopy (Invited Paper) [8785-288]

J. Garcia-Sucerquia, Univ. Nacional de Colombia Sede Medellín (Colombia)

878573 Illumination analysis of the digital pattern recognition system by Bessel masks and onedimensional signatures [8785-688]

S. Solorza, Univ. Autónoma de Baja California (Mexico); J. Álvarez-Borrego, Ctr. de Investigación Científica y de Educación Superior de Ensenada B.C. (Mexico)

\section{POSTER SESSION II}

878574 Photon kinetic description of nonlinear cross-phase modulation [8785-375]

J. T. Mendonça, Instituto Superior Técnico (Portugal); H. Crespo, Univ. do Porto (Portugal);

A. A. Amorim, Univ. do Porto (Portugal) and Instituto Politécnico do Porto (Portugal);

L. M. Bernardo, Univ. do Porto (Portugal)

878575 Numerical evaluation of the intensity transport equation for well-known wavefronts and intensity distributions [8785-378]

M. Campos-García, Univ. Nacional Autónoma de México (Mexico); F. Granados-Agustín,

A. Cornejo-Rodríguez, Instituto Nacional de Astrofísica, Óptica y Electrónica (Mexico);

A. Estrada-Molina, M. Avendaño-Alejo, Univ. Nacional Autónoma de México (Mexico);

V. I. Moreno-Oliva, Univ. del Istmo (Mexico)

$87857 \mathrm{~A}$ Optical characterization of volcanic ash using diffuse reflection spectroscopy [8785-393]

D. K. Bravo, N. Falcón, F. J. Narea, R. A. Muñoz, A. A. Muñoz, Univ. de Carabobo (Venezuela)

87857 B New formulation of the laws of reflection of light [8785-394]

Á. L. Pérez, G. Martínez, M. I. Suero, Univ. de Extremadura (Spain)

$87857 \mathrm{~F}$ Symmetries in the frequency space of the four-wave mixing signal [8785-40]

J. L. Paz, A. Mastrodomenico, M. A. Izquierdo, Univ. Simón Bolívar (Venezvela)

$87857 \mathrm{G}$ Estimation of hygroscopic power of electrotechnical materials by dynamic speckle technique [8785-401]

G. Bertolini, C. I. Cabello, CINDECA (Argentina); R. Arizaga, M. Trivi, Ctr. de Investigaciones Ópticas (Argentina) and Univ. Nacional de la Plata (Argentina); G. Barbera, Univ. Nacional de la Plata (Argentina)

$8785 \mathrm{7H}$ Automated coal petrography for macerals characterization using histograms technique [8785-406]

J. R. Armenta Mejía, Univ. de La Guajira (Colombia); L. Mattos, C. O. Torres, Univ. Popular del Cesar (Colombia)

$87857 \mathrm{~K}$ Design of a control system for interferometric fringe stabilization system with remote access [8785-412]

J. Espitia-Gómez, L. Ángel-Toro, D. Velásquez, Univ. EAFIT (Colombia) 
$87857 \mathrm{~T}$ Spectroscopy and microscopy characterization of spiropyran auto-assemble device [8785-424]

A. Ortiz Ramírez, R. Delgado Macuil, P. Zaca Moran, M. Rojas López, Ctr. de Investigación en Biotecnología Aplicada (Mexico)

8785 7W Obtaining circularly polarized highly focused fields: a proposal [8785-43]

D. Maluenda, Univ. de Barcelona (Spain); R. Martínez-Herrero, Univ. Complutense de Madrid (Spain); I. Juvells, A. Carnicer, Univ. de Barcelona (Spain)

$87857 Y \quad$ New method for sub-structured Ronchi rulings generation and his irradiance profile [8785-431]

D. Aguirre-Aguirre, F. S. Granados-Agustín, B. Villalobos-Mendoza, R. Izazaga-Pérez, Instituto Nacional de Astrofísica, Óptica y Electrónica (Mexico); M. Campos-García, Univ. Nacional Autónoma de México (Mexico); A. Cornejo-Rodríguez, Instituto Nacional de Astrofísica, Óptica y Electrónica (Mexico)

$878581 \quad$ Liquid crystal display for phase shifting [8785-435]

B. Villalobos-Mendoza, F. S. Granados-Agustín, D. Aguirre-Aguirre, A. Cornejo-Rodríguez, Instituto Nacional de Astrofísica, Óptica y Electrónica (Mexico)

878587 The optical nonlinearity of gold nanoparticles prepared by bioreduction method [8785-445] A. Balbuena Ortega, M. L. Arroyo Carrasco, Benemérita Univ. Autónoma de Puebla (Mexico); V. L. Gayou, A. Orduña Díaz, R. Delgado Macuil, M. Rojas López, Ctr. de Investigación en Biotecnología Aplicada (Mexico)

$878589 \quad$ Null Ronchi-Hartmann test for an aspheric concave mirror [8785-448] M. Avendaño-Alejo, G. Santiago-Castillo, M. Campos-García, Univ. Nacional Autónoma de México (Mexico); I. Moreno-Oliva, Univ. del Istmo (Mexico); R. Díaz-Uribe, Univ. Nacional Autónoma de México (Mexico)

8785 8A Optimization of irradiation patterns using light emitting diodes [8785-449]

E. Rojas, S. Stolik, J. M. de la Rosa, Instituto Politécnico Nacional (Mexico); M. Pérez Maldonado, Univ. de La Habana (Cuba)

$87858 \mathrm{C}$ Effect of the internal modulation of speckles in displacement measurements in optical vortex metrology [8785-452]

D. Gomez-Tejada, Univ. EAFIT (Colombia) and Univ. Nacional de Colombia (Colombia);

D. Sierra-Sosa, Univ. EAFIT (Colombia) and Ctr. de Investigaciones Ópticas (Argentina);

R. Castañeda, Univ. Nacional de Colombia Sede Medellín (Colombia);

L. Angel-Toro, Univ. EAFIT (Colombia)

$87858 \mathrm{~F} \quad$ Optical and electrical characterization of CdS-Glycine thin films with ammonia free buffer grown at different temperatures for solar cells applications [8785-459]

D. Berman-Mendoza, D. Quiñones-Urías, S. Ferra-González, A. Vera-Marquina,

A. Rojas-Hernández, R. Gómez Fuentes, A. García-Juárez, A. L. Leal-Cruz,

A. Ramos-Carrasco, Univ. de Sonora (Mexico)

$87858 \mathrm{G}$ Quantum teleportation using bipartite entangled photon-added non-linear coherent states [8785-46]

E. Castro, E. J. Páez, C. L. Ladera, A. Zambrano, Univ. Simón Bolívar (Venezuela) 
878581 Optical stimulated thermoluminescence in silicon rich oxide nanostructured films [8785-461]

D. Berman-Mendoza, Univ. de Sonora (Mexico); M. Aceves-Mijares, Instituto Nacional de Astrofísica, Óptica y Electrónica (Mexico); T. Piters, R. García-Gutiérrez, C. F. Ruiz-Valdez, Univ. de Sonora (Mexico); A. Ramos-Carrasco, Ctr. de Investigación en Materiales Avanzados, S.C. (Mexico); L. R. Berriel-Valdos, Instituto Nacional de Astrofísica, Óptica y Electrónica (Mexico)

$87858 \mathrm{M}$ Study and characterization of laser spot for plasma generation [8785-471]

A. Quiroga, P. Pacheco, R. Sarmiento, F. Racedo, Univ. del Atlantico (Colombia)

$87858 \mathrm{P}$ Testing the AUDI2000 colour-difference formula for solid colours using some visual datasets with usefulness to automotive industry [8785-479]

J. Martínez-García, M. Melgosa, L. Gómez-Robledo, Univ. of Granada (Spain); C. Li, Univ. of Science and Technology Liaoning (China); M. Huang, H. Liu, Beijing Institute of Graphic Communication (China); G. Cui, Wenzhou Univ. (China); M. R. Luo, Univ. of Leeds (United Kingdom) and Zhejiang Univ. (China); T. Dauser, AUDI AG (Germany)

$87858 Q \quad$ Quantum discord of bipartite entangled non-linear coherent states [8785-48] E. Castro, A. Zambrano, C. L. Ladera, R. Gómez, Univ. Simón Bolívar (Venezuela)

$87858 \mathrm{U}$ Quantum discord with weak measurement operators of quasi-Werner states based on bipartite entangled coherent states [8785-49]

E. Castro, R. Gómez, C. L. Ladera, A. Zambrano, Univ. Simón Bolívar (Venezuela)

$87858 \mathrm{~V} \quad$ Digital image quality measurements by objective and subjective methods from series of parametrically degraded images [8785-496]

A. Tachó, C. Mitjà, B. Martínez, J. Escofet, M. Ralló, Univ. Politècnica de Catalunya (Spain)

$87858 \mathrm{~W}$ Angular distribution of the averaged luminous intensity of low power LEDs transfer standards [8785-497]

J. L. Velázquez, A. Ferrero, Instituto de Óptica "Daza de Valdés" (Spain); M. López, Physikalisch-Technische Bundesanstalt (Germany); A. Pons, A. Villamarín, J. Campos, Instituto de Óptica "Daza de Valdés" (Spain); A. Sperling, Physikalisch-Technische Bundesanstalt (Germany)

$87858 \mathrm{X}$ Automated system for acquisition and image processing for the control and monitoring boned nopal [8785-5]

E. Luevano, E. de Posada, M. Arronte, L. Ponce, T. Flores, Ctr. de Investigación en Ciencia Aplicada y Tecnología Avanzada (Mexico)

878593 Comparative study on compact planar waveguide based photonic integrated couplers using simple effective index method [8785-516]

B. Deka, Girijananda Chowdhury Institute of Management and Technology (India) and Tezpur Univ. (India); A. Dutta, P. P. Sahu, Tezpur Univ. (India)

878594 Measurement of the r63 electrooptic constant of a KDP crystal using weak quantum measurement [8785-52]

A. Guzmán, C. Ladera, E. Castro, Univ. Simón Bolívar (Venezuela) 
878597 Study of measurement and calculation of the relative amplitude of accommodation [8785-525]

P. Coloma, I. Pascual, D. de Fez, V. Camps, Univ. de Alicante (Spain)

878591 Single-layer electroluminescent devices based on fluorene-1H-pyrazolo[3,4-b]quinoxaline co-polymers [8785-552]

M. Pokladko-Kowar, Cracow Univ. of Technology (Poland); A. Danel, Ł. Chacaga,

Agricultural Univ. of Cracow (Poland)

$87859 \mathrm{M}$ Panoramic fringe projection by using a conical mirror [8785-57]

V. H. Flores, Ctr. de Investigaciones en Óptica, A.C. (Mexico); L. Casaletto, K. Genovese,

Univ. degli Studi della Basilicata (Italy); J. A. Rayas, A. Martinez, Ctr. de Investigaciones en

Óptica, A.C. (Mexico)

$87859 \mathrm{~S}$ Optical bandgap enhancement of $\mathrm{a}-\mathrm{SiC}$ through hydrogen incorporation and thermal annealing treatments [8785-584]

L. M. Montañez, Pontificia Univ. Católica del Perú (Peru); J. A. Guerra, Pontificia Univ. Católica del Perú (Peru) and Friedrich-Alexander-Univ. Erlangen-Nürnberg (Germany); K. Zegarra, Pontificia Univ. Católica del Perú (Peru); S. Kreppel, Friedrich-Alexander-Univ. Erlangen-Nürnberg (Germany); F. De Zela, Pontificia Univ. Católica del Perú (Peru); A. Winnacker, Friedrich-Alexander-Univ. Erlangen-Nürnberg (Germany); R. Weingärtner, Pontificia Univ. Católica del Perú (Peru) and Friedrich-Alexander-Univ. Erlangen-Nürnberg (Germany)

87859 9Y Comparative study of Cr:Nd:YAG and Nd:YAG solar laser performances [8785-597]

D. Liang, J. Almeida, D. Garcia, Univ. Nova de Lisboa (Portugal)

$87859 Z$ Top lateral refraction and reflection of polarized light in lenses, coplanar lens system: applications [8785-598]

L. J. Miranda Díaz, Ctr. de Aplicaciones Tecnologicas y Desarrollo Nuclear (Cuba)

8785 A5 Non-invasive topographic inspection of wood artwork [8785-621]

M. F. M. Costa, Univ. do Minho (Portugal)

8785 A7 Digital holographic interferometry accelerated with GPU: application in mechanical microdeformation measurement operating at video rate (Best Student Paper Award) [8785-64]

N. Múnera Ortiz, C. A. Trujillo, J. García-Sucerquia, Univ. Nacional de Colombia Sede Medellín (Colombia)

8785 A9 Spectrum photoluminescence measuring system of porous silicon samples [8785-65] T. F. Paes, A. F. Beloto, L. A. Berni, L. M. Silva, Instituto Nacional de Pesquisas Espaciais (Brazil)

$8785 \mathrm{AB}$ Overall characterization of the polarization structure of radially polarized partially coherent vortex beams [8785-66]

R. Martinez-Herrero, F. Prado, Univ. Complutense de Madrid (Spain)

$8785 \mathrm{AC} \quad$ Measuring the area of tear film break-up by image analysis software [8785-679]

H. Pena-Verdeal, C. García-Resúa, Univ. de Santiago de Compostela (Spain); L. Ramos, Univ. da Coruña (Spain); A. Mosquera, E. Yebra-Pimentel, M. J. Giráldez, Univ. de Santiago de Compostela (Spain) 
$8785 \mathrm{AE} \quad$ Interobserver and intraobserver repeatability of lipid layer pattern evaluation by two experienced observers [8785-680]

C. García-Resúa, H. Pena-Verdeal, Univ. de Santiago de Compostela (Spain); M. Lira, Univ. do Minho (Portugal); M. G. Penedo, Univ. da Coruña (Spain); M. J. Giráldez,

E. Yebra-Pimentel, Univ. de Santiago de Compostela (Spain)

$8785 \mathrm{Al}$ Variation of spatial soliton in liquid crystals due change of polarization of the incident laser beam [8785-70]

A. A. Rodríguez-Rosales, C. J. Román Moreno, R. Ortega-Martínez, Univ. Nacional Autónoma de México (Mexico)

8785 AJ Analysis of the stability of an active mode-locking pulsed laser for ultra-short pulses generation [8785-71]

Y. E. Bracamontes Rodríguez, G. Beltrán Pérez, E. Kuzin, J. Castillo Mixcóatl,

S. Muñoz Aguirre, Benemérita Univ. Autónoma de Puebla (Mexico)

8785 AK Optimization of a Fabry-Perot Q-switch fiber optic laser [8785-74]

I. Armas Rivera, G. Beltrán Pérez, E. Kuzin, J. Castillo Mixcóatl, S. Muñoz Aguirre, Benemérita Univ. Autónoma de Puebla (Mexico)

$8785 \mathrm{AL} \quad$ Full model analysis of the four-wave mixing anti-STOKES component growth in the wavelength-division multiplexing solitons systems near the zero-dispersion wavelength [8785-75]

L. Mandeng Mandeng, S. Fewo Ibraid, C. Tchawoua, Univ. de Yaoundé 1 (Cameroon)

$8785 \mathrm{AM}$ Optical waveguides formed by carbon implantation on $\mathrm{SiO}_{2}$ [8785-79]

E. Flores-Romero, J. M. Zarate-Reyes, H. Silva-Pereyra, A. Oliver, L. Rodríguez-Fernández, Univ. Nacional Autónoma de México (Mexico)

$8785 \mathrm{AQ}$ Optical properties of Nd:YAG crystals implanted with Ni and Ti ions [8785-86]

G. V. Vázquez, Ctr. de Investigaciones en Óptica, A.C. (Mexico); R. Trejo-Luna, Univ. Nacional Autónoma de México (Mexico); D. Ramírez, Ctr. de Investigaciones en Óptica, A.C. (Mexico); J. Rickards, Univ. Nacional Autónoma de México (Mexico)

8785 AR A 2D continuous wavelet transform method for InSAR phase-maps denoising [8785-87] N. Escalante, J. Villa, I. De la Rosa, C. Olvera-Olvera, D. Alaniz-Lumbreras,

O. Gutierrez-Mata, Univ. Autónoma de Zacatecas (Mexico)

8785 AT Band structure of a periodic waveguide that include a dispersive metamaterial [8785-91] A. Mendoza-Suárez, H. Pérez-Aguilar, Univ. Michoacana de San Nicolás de Hidalgo (Mexico)

$8785 \mathrm{AU}$ The perfect vortex and its realization by the liquid crystal spatial light modulator [8785-94] C. Rickenstorff, A. S. Ostrovsky, Univ. Autónoma de Puebla (Mexico)

8785 AW Skin-light interaction of three main chromofores in skin affected by Port Wine Stain [8785-97]

S. Mújica Ascencio, J. S. Velázquez González, J. A. Álvarez Chávez, Ctr. de Investigación e Innovación Tecnológica (Mexico) 
8785 AX Digital controller applied to phase control in photorefractive crystals [8785-99]

H. Lorduy G., Univ. Pontificia Bolivariana (Colombia); J. A. Valencia, Servicio Nacional de Aprendizaje (Colombia)

8785 AY Retro-diffusion and transmission of laser radiation to characterize the paper fiber distribution and mass density [8785-110]

A. de O. Mendes, P. T. Fiadeiro, A. P. Costa, M. E. Amaral, Univ. da Beira Interior (Portugal);

M. N. Belgacem, Institut National Polytechnique de Grenoble (France)

$8785 \mathrm{AZ} \quad$ UV-visible transmittance of silicone-hydrogel contact lenses measured with a fiber optic spectrometer [8785-179]

R. Fuentes, E. Fernández, I. Pascual, C. García, Univ. de Alicante (Spain)

\section{SESSION XIX}

8785 B3 Study on highly sensitive asymmetric waveguide optical sensor for detection of sucrose concentration based on leaky quasi-modes [8785-675]

A. Dutta, B. Deka, P. P. Sahu, Tezpur Univ. (India)

8785 B6 Abel transform inversion using Kalman filter [8785-154]

E. González-Ramírez, E. de la Rosa Miranda, J. G. Arceo-Olague, J. J. Villa-Hernández,

I. de la Rosa Vargas, T. Saucedo Anaya, Univ. Autónoma de Zacatecas (Mexico);

L. R. Berriel-Valdos, Instituto Nacional de Astrofísica, Óptica y Electrónica (Mexico);

M. A. Araiza-Esquivel, Univ. Autónoma de Zacatecas (Mexico)

\section{SESSION XX}

8785 B9 Remote access to an interferometric fringes stabilization active system via RENATA [8785-693]

J. Espitia-Gómez, L. Ángel-Toro, Univ. EAFIT (Colombia)

8785 BA Phase unwrapping using Chebyshev polynomials [8785-158]

E. de la Rosa Miranda, E. González-Ramírez, J. G. Arceo-Olague, J. J. Villa-Hernández,

I. de la Rosa Vargas, T. Saucedo-Anaya, Univ. Autónoma de Zacatecas (Mexico);

L. R. Berriel-Valdos, Instituto Nacional de Astrofísica, Óptica y Electrónica (Mexico);

N. Escalante, Univ. Autónoma de Zacatecas (Mexico)

\section{SESSION XXI}

8785 BD Optimization of digitization procedures in cultural heritage preservation [8785-495]

B. Martínez, C. Mitjà, J. Escofet, Univ. Politècnica de Catalunya (Spain)

8785 BF Laser removal of graffiti from Pink Morelia Quarry [8785-300]

J. Penide, F. Quintero, Univ. de Vigo (Spain); A. Riveiro, Ctr. Univ. de la Defensa (Spain);

A. Sánchez-Castillo, Univ. Michoacana de San Nicolás de Hidalgo (Mexico); R. Comesaña,

J. del Val, F. Lusquiños, J. Pou, Univ. de Vigo (Spain) 
$8785 \mathrm{BH} \quad$ Fiber specklegram sensors (FSS) for measuring high frequency mechanical perturbations [8785-250]

A. Hoyos, Politécnico Colombiano Jaime Isaza Cadavid (Colombia); N. D. Gómez, Instituto Tecnológico Metropolitano (Colombia); J. A. Gómez, Politécnico Colombiano Jaime Isaza Cadavid (Colombia)

$8785 \mathrm{BI}$ Single frame method for resolution enhancement of the Fourier fringe analysis method [8785-553]

P. J. Tavares, Prudente and Tavares (Portugal); M. A. P. Vaz, Univ. do Porto (Portugal)

$8785 \mathrm{BL} \quad$ High accuracy piston and wobble sensor based on heterodyne interferometry [8785-328]

C. Silva, A. Cabral, M. Abreu, J. M. Rebordão, D. Castro Alves, Univ. de Lisboa (Portugal)

\section{SESSION XXIII}

8785 BP Spatially inhomogeneous phase-modulating interferometry by non-quadrature amplitude modulation [8785-167]

U. Rivera-Ortega, C. Meneses-Fabian, G. Rodriguez-Zurita, Benemérita Univ. Autónoma de Puebla (Mexico)

\section{Part Three}

\section{SESSION XXIV}

8785 BU Nonequilibrium free diffusion in seed leachate [8785-604]

L. Ortiz G., P. Riquelme P., R. Guzmán, Univ. de la Frontera (Chile) and Univ. de Concepción (Chile)

8785 BV Study of the ink-paper interaction by image analysis: surface and bulk inspection [8785-124]

P. T. Fiadeiro, A. de O. Mendes, A. M. M. Ramos, S. C. L. de Sousa, Univ. da Beira Interior (Portugal)

8785 BW Sensitivity enhancement of evanescent waveguide optical sensor for detecting adulterant traces in petroleum products using SiON technology [8785-322]

A. Dutta, B. Deka, P. P. Sahu, Tezpur Univ. (India)

8785 BX Study on compactness of planar waveguide based integrated optic couplers using tooth shaped grating assisted geometry [8785-676]

B. Deka, Girijananda Chowdhury Institute of Management and Technology (India) and

Tezpur Univ. (India); A. Dutta, P. P. Sahu, Tezpur Univ. (India) 
8785 BZ Remote fiber sensors and optical amplification (Invited Paper) [8785-297]

M. J. Pontes, Univ. Federal do Espirito Santo (Brazil); T. V. N. Coelho, Univ. Federal de Juiz de Fora (Brazil); J. P. Carvalho, J. L. Santos, A. Guerreiro, INESC Porto (Portugal)

8785 C2 Gain flattening analysis for Raman+EDFA hybrid amplifiers using recycled pump power for WDM systems [8785-686]

M. M. Jardim Martini, Ctr. Federal de Educação Tecnológica de Minas Gerais (Brazil);

C. E. S. Castellani, M. J. Pontes, M. R. N. Ribeiro, Univ. Federal do Espírito Santo (Brazil);

H. J. Kalinowski, Univ. Tecnológica Federal do Paraná (Brazil)

8785 C8 Fabrication of polymer Fabry-Perot fiber sensors using optical fiber microheaters (Invited Paper) [8785-42]

M. S. Cano-Velázquez, J. Hernández-Cordero, Univ. Nacional Autónoma de México (Mexico)

8785 C9 Laser processed preforms for microstructured optical fibers [8785-117]

M. Becker, Institut für Photonische Technologien e.V. (Germany); M. Werner, O. Fitzau,

D. Esser, Fraunhofer-Institut für Lasertechnik (Germany); J. Kobelke, K. Schuster, A. Lorenz,

A. Schwuchow, M. Rothhardt, Institut für Photonische Technologien e.V. (Germany);

D. Hoffmann, Fraunhofer-Institut für Lasertechnik (Germany); H. Bartelt, Institut für

Photonische Technologien e.V. (Germany)

8785 CC Study of temperature sensor fiber with high germanium doped core [8785-278]

F. C. Fávero, M. Becker, R. Spittel, M. Rothhardt, J. Kobelke, H. Bartelt, Institut für

Photonische Technologien e.V. (Germany)

SESSION XXV

$8785 \mathrm{CF}$ Interference with electrons: from thought to real experiments (Invited Paper) [8785-141]

G. Matteucci, Univ. degli Studi di Bologna (Italy)

8785 CG Enabling quantum communications through accurate photons polarization control [8785-305]

Á. J. Almeida, N. J. Muga, N. A. Silva, Univ. de Aveiro (Portugal) and Instituto de Telecomunicações (Portugal); A. D. Stojanovic, Instituto de Telecomunicações (Portugal); P. S. André, A. N. Pinto, Univ. de Aveiro (Portugal) and Instituto de Telecomunicações (Portugal); J. Mora, J. Capmany, Univ. Politècnica de València (Spain)

$8785 \mathrm{Cl}$ Pump and filtering optimization in Mamyshev regenerator [8785-355]

A. Supe, Riga Technical Univ. (Latvia); G. M. Fernandes, N. J. Muga, A. N. Pinto, Instituto de Telecomunicações (Portugal) and Univ. de Aveiro (Portugal); M. F. S. Ferreira, Univ. de Aveiro (Portugal) 
$8785 \mathrm{CL}$ Correlation applied to the recognition of regular geometric figures [8785-329]

W. Lasso, Y. Morales, F. Vega, L. Díaz, Univ. Popular del Cesar (Colombia); D. Flórez, Univ. del Zulia (Venezuela); C. Torres, Univ. Popular del Cesar (Colombia)

\section{SESSION XXVII}

$8785 \mathrm{CO}$ Ophthalmic compensation of visual ametropia based on a programmable diffractive lens [8785-150]

M. S. Millán, E. Pérez-Cabré, L. A. Romero, N. Ramírez, Univ. Politècnica de Catalunya (Spain)

$8785 \mathrm{CP}$ Experiment design for through-focus testing of intraocular lenses [8785-151]

M. S. Millán, F. Alba-Bueno, F. Vega, Univ. Politècnica de Catalunya (Spain)

8785 CS Development of an optical simulator of the human eye [8785-284]

J. M. P. Coelho, Univ. de Lisboa (Portugal); A. Baião, Univ. de Lisboa (Portugal) and Univ.

Nova de Lisboa (Portugal); P. Vieira, Univ. Nova de Lisboa (Portugal)

\section{SESSION XXVIII}

$8785 \mathrm{CU}$ Laser scanners: from industrial to biomedical applications [8785-131]

V.-F. Duma, Aurel Vlaicu Univ. of Arad (Romania)

$8785 \mathrm{CX}$ Optical characterization of thermal properties of biological tissue [8785-215]

A. Gutierrez-Arroyo, C. Sánchez Pérez, N. Alemán-García, C. Piña-Barba, Univ. Nacional

Autónoma de México (Mexico)

$8785 \mathrm{CY} \quad$ PIV and digital holography for measuring blood flows and vessel wall dynamics [8785-311]

L. Arévalo, E. Roche, V. Palero, M. A. Martínez, M. P. Arroyo, Univ. de Zaragoza (Spain)

\section{POSTER SESSION III}

$8785 \mathrm{CZ}$ Power measuring in ophthalmic lenses using lateral amplification [8785-103]

P. G. Mendoza-Villegas, G. Trujillo-Schiaffino, D. P. Salas-Peimbert, M. Anguiano-Morales,

L. F. Corral-Martinez, Instituto Technologico de Chihuahua (Mexico)

8785 D0 Sphere power measuring in ophthalmic lenses by infinite fringe moiré deflectometry [8785-104]

J. A. García-Ruelas, G. Trujillo-Schiaffino, D. P. Salas-Peimbert, M. Anguiano-Morales,

L. F. Corral-Martinez, Instituto Technologico de Chihuahua (Mexico)

$8785 \mathrm{Dl} \quad$ Blind restoration of retinal images degraded by space-variant blur with adaptive blur estimation [8785-126]

A. G. Marrugo, Univ. Politècnica de Catalunya (Spain) and Univ. Tecnológica de Bolivar (Colombia); M. S. Millán, Univ. Politècnica de Catalunya (Spain); M. Šorel, F. Šroubek, Institute of Information Theory and Automation (Czech Republic) 
8785 D2 Detection of fungi colony growth on bones by dynamic speckle [8785-161]

F. M. Vincitorio, Univ. Tecnológica Nacional (Argentina); N. Budini, Univ. Tecnológica

Nacional (Argentina) and Instituto de Física del Litoral (Argentina); C. Mulone, M. Spector, C. Freyre, Univ. Tecnológica Nacional (Argentina); A. J. López Díaz, A. Ramil, Univ. da Coruña (Spain)

8785 D7 Diffuse reflectance spectroscopy and optical polarization imaging of in-vivo biological tissue [8785-216]

A. Mora-Núñez, Univ. de Guadalajara (Mexico); Y. Castillejos, Ctr. de Investigaciones en Óptica, A.C. (Mexico); G. García-Torales, Univ. de Guadalajara (Mexico);

G. Martínez-Ponce, Ctr. de Investigaciones en Óptica, A.C. (Mexico)

8785 DA Development of a low cost pupillometer-eyetracker and applications [8785-26]

A. Bianchetti, Univ. de Buenos Aires (Argentina); L. I. Perez, S. A. Comastri, Univ. de Buenos Aires (Argentina) and Consejo Nacional de Investigaciones Científicas y Técnicas (Argentina)

8785 DB GUCLF: a new light field face database [8785-290]

R. Raghavendra, K. B. Raja, B. Yang, C. Busch, Gjøvik Univ. College (Norway)

8785 DE Optical simulation of laser beam phase-shaping focusing optimization in biological tissues [8785-319]

R. Gomes, Univ. de Lisboa (Portugal); P. Vieira, Univ. Nova de Lisboa (Portugal);

J. M. P. Coelho, Univ. de Lisboa (Portugal)

8785 DF Parameterization using Fourier series expansion of the diffuse reflectance of human skin to vary the concentration of the melanocytes [8785-324]

J. F. Narea, A. A. Muñoz, Univ. de Carabobo (Venezuela); J. Castro, Instituto Nacional de Astrofísica, Óptica y Electrónica (Mexico); R. A. Muñoz, C. E. Villalba, M. F. Martinez,

K. D. Bravo, Univ. de Carabobo (Venezuela)

$8785 \mathrm{DH} \quad$ Dynamical speckles patterns of action potential transmission effects in squid giant axon membrane [8785-372]

J. J. Llovera-González, A. B. Moreno-Yeras, Instituto Superior Politécnico José Antonio

Echeverría (Cuba); M. Muramatsu, D. Soga, Univ. de São Paulo (Brazil); R. L. Serra-Toledo, Instituto Superior Politécnico José Antonio Echeverría (Cuba); D. S. F. Magalhães, Hospital de Clínicas da UNICAMP (Brazil)

$8785 \mathrm{DI} \quad$ Speckle patterns during the spreading of lung surfactant [8785-381]

J. J. Llovera-González, A. B. Moreno-Yeras, Instituto Superior Politécnico José Antonio Echeverría (Cuba); D. M. Martínez-Muñoz, M. Z. J. Ferreira, W. Shin Nishitani, A. B. Almeida, A. M. Alencar, M. Muramatsu, Univ. de São Paulo (Brazil); R. L. Serra-Toledo, Instituto Superior Politécnico José Antonio Echeverría (Cuba)

8785 DJ Parametrization histological grade white adipose tissue of the breast by the cubic spline interpolation [8785-388]

L. A. Martinez, Univ. de Carabobo (Venezuela); F. J. Narea, Univ. de Carabobo

(Venezuela) and Univ. Central de Venezuela (Venezuela); F. Cedeño, Univ. de Carabobo (Venezuela); A. A. Muñoz, Univ. de Carabobo (Venezuela) and Univ. Central de Venezuela (Venezuela); A. Reigosa, Univ. Central de Venezuela (Venezuela); K. Bravo, Univ. de Carabobo (Venezuela) 
8785 DN Experimental investigation of surface tension in Newtonian and non-Newtonian fluids with optical diffractometry [8785-398]

M. Zargham, Univ. of Zanjan (Iran, Islamic Republic of); A. Moradi, Univ. of Zanjan (Iran, Islamic Republic of) and Institute for Advanced Studies in Basic Sciences (Iran, Islamic Republic of); A. Najafi, Univ. of Zanjan (Iran, Islamic Republic of)

8785 DO Determination of quality parameters in apple 'Smoothee Golden Delicious' using backscattering laser imaging [8785-400]

V. Lafuente, Estación Experimental Aula Dei (Spain) and Univ. de Zaragoza (Spain); J. Val, Estación Experimental Aula Dei (Spain); C. Urzola, I. Negueruela, Univ. de Zaragoza (Spain)

8785 DR Gabor transform applied to superresolution in optical systems [8785-414]

C. Jimenez, R. Pérez, Univ. de la Gaujira (Colombia); C. O. Torres, Univ. Popular del Cesar (Colombia)

8785 DT Characterization of PET preforms using spectral domain optical coherence tomography [8785-491]

H. Hosseiny, INESC TEC (Portugal); M. J. Ferreira, EnerMeter - Sistemas de Medição, Lda (Portugal) and Univ. do Minho (Portugal); T. Martins, EnerMeter - Sistemas de Medição, Lda (Portugal); C. Carmelo Rosa, INESC TEC (Portugal)

8785 DU Low level laser therapy on injured rat muscle: assessment of irradiation parameters (Best Student Paper Award) [8785-61]

M. Mantineo, J. P. Pinheiro, A. M. Morgado, Univ. de Coimbra (Portugal)

8785 DW An irradiation system for photodynamic therapy with a fiber-optic sensor for measuring tissue oxygen [8785-76]

L. Quintanar, D. Fabila, S. Stolik, J. M. de la Rosa, Instituto Politécnico Nacional (Mexico)

8785 DY Low power laser and LED irradiation effect on proliferation and differentiation of Wistar rats mesenchymal stem cells [8785-83]

D. Mancera, E. Solarte, L. Fierro, W. Criollo, Univ. del Valle (Colombia)

8785 EO Gloss measurements and rugometric inspection in dental biomaterials [8785-145] A. Fernández-Oliveras, Univ. de Granada (Spain); M. F. M. Costa, Univ. do Minho (Portugal); A. Yebra, M. Rubiño, M. M. Pérez, Univ. de Granada (Spain)

$8785 \mathrm{El}$ Tuning a fast linear camera used within a Talbot bands spectrometer-based optical coherence tomography set-up [8785-174]

M. J. Marques, A. Bradu, A. G. Podoleanu, Univ. of Kent (United Kingdom)

8785 E2 Nonlinear correlation by using invariant identity vectors signatures to identify different species of fish [8785-196]

C. Fimbres-Castro, Univ. Autónoma de Baja California (Mexico); J. Álvarez-Borrego, Ctr. de Investigación Científica y de Educación Superior de Ensenada B.C. (Mexico)

8785 E3 Laser bioengineering of glass-titanium implants surface [8785-270]

F. Lusquiños, F. Arias-González, J. Penide, J. del Val, R. Comesaña, F. Quintero, Univ. de Vigo (Spain); A. Riveiro, Ctr. Univ. de la Defensa (Spain); M. Boutinguiza, Univ. de Vigo (Spain); M. J. Pascual, A. Durán, Instituto de Cerámica y Vidrio (Spain); J. Pou, Univ. de Vigo (Spain) 
8785 E4 Artifacts cancellation in Fourier domain optical coherence tomography using balance detection [8785-407]

A. Bradu, A. G. Podoleanu, Univ. of Kent (United Kingdom)

8785 E8 A diffuse reflectance spectroscopy system to study biological tissues [8785-456]

A. de la Cadena, S. Stolik, J. M. de la Rosa, Instituto Politécnico Nacional (Mexico)

8785 E9 Irradiation system for interstitial photodynamic therapy [8785-457]

L. Pacheco, S. Stolik, J. De la Rosa, Instituto Politécnico Nacional (Mexico)

8785 EA Design of matrix irradiation system for external tissue phototherapy with temperature control [8785-458]

F. Y. López S., S. Stolik Isakina, J. M. de La Rosa Vázquez, Instituto Politécnico Nacional (Mexico)

8785 EF Determination of optical properties in dental restorative biomaterials using the inverseadding-doubling method (Best Student Paper Award) [8785-492]

A. Fernández-Oliveras, M. Rubiño, M. M. Pérez, Univ. de Granada (Spain)

8785 EG Rugometric and microtopographic non-invasive inspection in dental-resin composites and zirconia ceramics [8785-51]

A. Fernández-Oliveras, Univ. de Granada (Spain); M. F. M. Costa, Univ. do Minho (Portugal); O. E. Pecho, M. Rubiño, M. M. Pérez, Univ. de Granada (Spain)

$8785 \mathrm{EH} \quad$ Evaluation of metal-polymeric fixed partial prosthesis using optical coherence tomography [8785-528]

C. Sinescu, M. L. Negrutiu, Univ. of Medicine and Pharmacy Victor Babes Timisoara (Romania); V. F. Duma, Aurel Vlaicu Univ. of Arad (Romania); C. Marcauteanu, F. I. Topala, M. Rominu, Univ. of Medicine and Pharmacy Victor Babes Timisoara (Romania); A. Bradu,

A. G. Podoleanu, Univ. of Kent (United Kingdom)

$8785 \mathrm{EL}$ Morphological analysis of Bacillux polymyxa colonies: digital image analysis in wide-field microscopy [8785-574]

L. M. Guerrero, A. Plata G., G. Rincón C., H. Gutiérrez A., A. Plata, Univ. Industrial de Santander (Colombia)

8785 ER Assessing the optical properties of the healthy and emmetropic eye with accommodation [8785-638]

C. M. Oliveira, S. Franco, Univ. do Minho (Portugal)

8785 ES Influence of the thermo-oxidative degradation on the chemical structure of contact lenses [8785-643]

M. Lira, C. Alves, G. Botelho, Univ. do Minho (Portugal)

8785 ET Accuracy of two osmometers on standard samples: electrical impedance technique and freezing point depression technique [8785-677]

C. García-Resúa, H. Pena-Verdeal, M. Miñones, J. Gilino, M. J. Giraldez, E. Yebra-Pimentel, Univ. de Santiago de Compostela (Spain) 
8785 EU Criteria for lipid layer pattern evaluation: Pli-marker database [8785-678]

C. García-Resúa, H. Pena-Verdeal, Univ. de Santiago de Compostela (Spain);

B. Remeseiro, M. G. Penedo, Univ. of A Coruña (Spain); M. J. Giráldez, E. Yebra-Pimentel, Univ. de Santiago de Compostela (Spain)

$8785 \mathrm{EV} \quad$ Comparison between three methods to value lower tear meniscus measured by image software [8785-681]

C. García-Resúa, H. Pena-Verdeal, Univ. de Santiago de Compostela (Spain); M. Lira, M. E. R. Oliveira, Univ. do Minho (Portugal); M. J. Giráldez, E. Yebra-Pimentel, Univ. de Santiago de Compostela (Spain)

\section{POSTER SESSON XI SEONS}

8785 EX Optical fibre monitoring of Madeira wine estufagem process [8785-142]

C. Novo, Univ. de Aveiro (Portugal); L. Bilro, Instituto de Telecomunicações (Portugal); R. Ferreira, Univ. de Aveiro (Portugal); N. Alberto, Instituto de Telecomunicações (Portugal) and Univ. de Aveiro (Portugal); P. Antunes, Univ. de Aveiro (Portugal) and Instituto de Telecomunicações (Portugal); R. Nogueira, Instituto de Telecomunicações (Portugal); J. L. Pinto, Univ. de Aveiro (Portugal) and Instituto de Telecomunicações (Portugal)

$8785 \mathrm{EY} \quad$ A long period grating-based platform for the detection of E. coli proteins [8785-200] R. B. Queirós, INESC Porto (Portugal), Univ. do Porto (Portugal), and Instituto Politécnico do Porto (Portugal); C. Gouveia, INESC Porto (Portugal); J. R. A. Fernandes, Univ. de Trás-osMontes e Alto Douro (Portugal); P. V. S. Marques, INESC Porto (Portugal) and Univ. do Porto (Portugal); J. P. Noronha, Univ. Nova de Lisboa (Portugal); M. G. F. Sales, Instituto Politécnico do Porto (Portugal); P. A. S. Jorge, INESC Porto (Portugal)

8785 F0 Characterization of a fiber based heralded single photon source at telecom wavelength [8785-303]

N. A. Silva, A. N. Pinto, Univ. de Aveiro (Portugal) and Instituto de Telecomunicações (Portugal)

8785 F3 Wavelength-shift-free Mamyshev regenerator [8785-348]

G. M. Fernandes, Instituto de Telecomunicações (Portugal) and Univ. de Aveiro (Portugal); B. Tiburcio, Instituto de Telecomunicações (Portugal); N. J. Muga, A. N. Pinto, Instituto de Telecomunicações (Portugal) and Univ. de Aveiro (Portugal)

8785 F4 Nonlinear polarizers in low-birefringence optical fibers [8785-403] N. J. Muga, G. M. Fernandes, A. N. Pinto, Instituto de Telecomunicações (Portugal) and Univ. de Aveiro (Portugal)

8785 F5 Validation of dose measurements by scintillating fiber optic dosimeters for medical applications [8785-488]

A. Correia, INESC Porto (Portugal); R. Pirraco, IPOFG Porto (Portugal); C. C. Rosa, INESC Porto (Portugal) and Univ. do Porto (Portugal)

8785 F9 New developments on the design and modeling of fiber optical tweezers [8785-519] R. S. Rodrigues Ribeiro, INESC Porto (Portugal) and Univ. do Porto (Portugal); P. A. S. Jorge, Univ. do Porto (Portugal); A. Guerreiro, INESC Porto (Portugal) and Univ. do Porto (Portugal) 
8785 FA Design and characterization of a plastic optical fiber pH sensor [8785-530]

L. Ferreira, P. Simões, R. S. Carvalho, P. Lopes, M. Ferreira, Univ. de Aveiro (Portugal)

8785 FB Figure-of-eight cavity fiber laser based torsion sensor [8785-531]

M. S. Ferreira, J. L. Santos, INESC Porto (Portugal) and Univ. do Porto (Portugal); P. Mergo, Maria Curie-Sklodowska Univ. (Poland); O. Frazão, INESC Porto (Portugal)

$8785 \mathrm{FC}$ Characterization of a novel dissolved $\mathrm{CO}_{2}$ sensor for utilization in environmental monitoring and aquaculture industry [8785-535]

K. Balogh, INESC Porto (Portugal) and Univ. of Pécs (Hungary); J. M. Jesus, CIIMAR (Portugal); C. Gouveia, INESC Porto (Portugal) and Univ. da Madeira (Portugal);

J. O. Domingues, CIIMAR (Portugal); A. Markovics, Univ. of Pécs (Hungary); J. M. Baptista, Univ. da Madeira (Portugal); B. Kovacs, Univ. of Pécs (Hungary); C. M. Pereira, Univ. do Porto (Portugal); M.-T. Borges, CIIMAR (Portugal) and Univ. do Porto (Portugal);

P. A. S. Jorge, INESC Porto (Portugal)

$8785 \mathrm{FE} \quad$ High refractive index and temperature sensitivity LPGs for high temperature operation [8785-551]

I. M. Nascimento, INESC Porto (Portugal) and Univ. do Porto (Portugal); C. Gouveia, INESC Porto (Portugal) and Univ. da Madeira (Portugal); S. Jana, S. Bera, Central Glass and Ceramic Research Institute (India); J. M. Baptista, INESC Porto (Portugal) and Univ. da Madeira (Portugal); P. Moreira, INESC Porto (Portugal); P. Biwas, S. Bandyopadhyay, Central Glass and Ceramic Research Institute (India); P. A. S. Jorge, INESC Porto (Portugal)

$8785 \mathrm{FF} \quad$ Extremely small-core photonic crystal fiber fusion splicing with a single-mode fiber [8785-559]

B. D. Tiburcio, Instituto de Telecomunicações (Portugal); G. M. Fernandes, A. N. Pinto, Instituto de Telecomunicações (Portugal) and Univ. de Aveiro (Portugal)

$8785 \mathrm{FH} \quad$ Low-cost temperature measurement using LPG and total transmitted power [8785-564] P. Simões, P. Lopes, Univ. de Aveiro (Portugal)

$8785 \mathrm{FI} \quad$ Spatial soliton dynamics in cubic-quintic media [8785-572]

N. A. Silva, INESC TEC - UOSE (Portugal) and Univ. do Porto (Portugal); M. I. Carvalho, Univ. do Porto (Portugal) and INESC TEC - UTM (Portugal); A. Guerreiro, INESC TEC - UOSE (Portugal) and Univ. do Porto (Portugal)

8785 FJ Lightons: phonons with light [8785-575] N. A. Silva, INESC TEC - UOSE (Portugal) and Univ. do Porto (Portugal); M. I. Carvalho, Univ. do Porto (Portugal) and INESC TEC - UTM (Portugal); A. Guerreiro, INESC TEC - UOSE (Portugal) and Univ. do Porto (Portugal)

8785 FK SPR sensing with bimetallic layers in optical fibers and phase interrogation [8785-576] H. Moayyed, I. T. Leite, L. Coelho, J. L. Santos, INESC Porto (Portugal) and Univ. do Porto (Portugal); D. Viegas, INESC Porto (Portugal) and Cabelte, SA (Portugal) 
$8785 \mathrm{FL} \quad$ Ag-nanowire metamaterials: spectral reflectance analysis and homogenization models [8785-581]

I. T. Leite, INESC TEC (Portugal); A. O. Silva, INESC TEC (Portugal), Applied Electromagnetics Lab. (Brazil), and Univ. Federal do Pará (Brazil); A. Hierro-Rodríguez, INESC TEC (Portugal) and Univ. do Porto (Portugal); C. T. Sousa, M. P. Fernández-García, J. M. Teixeira, J. P. Araujo, Univ. do Porto (Portugal); M. T. Giraldi, Instituto Militar de Engenharia (Brazil); J. W. C. A. Costa, Applied Electromagnetics Lab. (Brazil) and Univ. Federal do Pará (Brazil); D. Viegas, INESC Porto (Portugal) and Cabelte, SA (Portugal); P. A. S. Jorge, INESC Porto (Portugal); A. Guerreiro, INESC Porto (Portugal) and Univ. do Porto (Portugal)

8785 FM Enhancement of refractive index sensitivity of the in-line Mach-Zehnder interferometer through bending [8785-635]

C. R. da Silveira, J. W. A. Costa, Univ. Federal do Pará (Brazil); M. T. R. Giraldi, Instituto Militar de Engenharia (Brazil); M. A. R. Franco, Instituto de Estudos Avançados (Brazil);

P. A. S. Jorge, INESC Porto (Portugal)

8785 FP Development of an electrical current sensor prototype for applications in high-power lines [8785-691]

I. M. Nascimento, INESC Porto (Portugal) and Univ. do Porto (Portugal); A. C. S. Brígida, INESC Porto (Portugal) and Univ. Federal do Pará (Portugal); J. M. Baptista, INESC Porto (Portugal) and Univ. da Madeira (Portugal); J. C. W. A. Costa, Univ. Federal do Pará (Brazil); M. A. G. Martinez, CEFET/RJ (Brazil); P. A. S. Jorge, INESC Porto (Portugal)

$8785 \mathrm{FQ}$ Monitoring of non-homogeneous strains in wood glued joints with embedded FBG optical sensors in mode I delamination tests [8785-694]

R. S. Maciel, O. Frazão, INESC TEC (Portugal); J. J. L. Morais, Univ. de Trás-os-Montes e Alto Douro (Portugal); J. R. A. Fernandes, INESC TEC (Portugal) and Univ. de Trás-os-Montes e Alto Douro (Portugal)

\section{SESSION XXIX}

$8785 \mathrm{FT}$ The influence of humidity, temperature, and oral contraceptive in tear [8785-282] R. A. R. C. Sousa, T. L. C. Ribeiro, Clinical Practice (Portugal); S. M. B. Moreira, Escola Secundária Vieira do Minho (Portugal); A. M. G. Baptista, Univ. do Minho (Portugal)

$8785 \mathrm{FW}$ The macular photostress test in diabetes, glaucoma, and cataract [8785-317] A. M. G. Baptista, Univ. do Minho (Portugal); R. A. R. C. Sousa, F. A. S. Q. Rocha, P. S. Fernandes, Clinical Practice (Portugal); A. F. Macedo, Univ. do Minho (Portugal)

\section{SESSION XXX}

$8785 \mathrm{Gl} \quad$ Biospeckle: fractional Fourier transform point of view [8785-338]

W. Lasso, Univ. Popular del Cesar (Colombia); E. A. Pérez Calderón, N. P. Castillo Serrano, Univ. del Zulia (Venezuela); L. Díaz M., C. O. Torres, Univ. Popular del Cesar (Colombia) 
8785 G2 A review on self-reference wavefront methods in optical testing (Plenary Paper) [8785-82] A. Cornejo-Rodríguez, F. S. Granados-Agustín, Instituto Nacional de Astrofísica, Óptica y Electrónica (Mexico)

\section{SESSION XXXI}

8785 G5 Emmetropic eyes: objective performance and clinical reference (Invited Paper) [8785-609] E. Tepichín-Rodríguez, A. S. Cruz Felix, E. López-Olazagasti, S. Balderas-Mata, Instituto Nacional de Astrofísica, Óptica y Electrónica (Mexico)

8785 G6 Development of a subjective refraction simulator [8785-371]

S. Perches, J. Ares, M. V. Collados, Univ. de Zaragoza (Spain)

\section{SESSION XXXII}

8785 GA Detection of the effect of nanoparticles on myelin figures growth using a compact digital holographic microscope [8785-339]

S. Ebrahimi, P. Soltani, Univ. of Zanjan (Iran, Islamic Republic of); A.-R. Moradi, Univ. of Zanjan (Iran, Islamic Republic of) and Institute for Advanced Studies in Basic Sciences (Iran, Islamic Republic of); L. Tayebi, Oklahoma State Univ. (United States)

8785 GB An ultraviolet intercomparison for medical equipment evaluation [8785-364] A. F. G. Ferreira Jr., R. Leone Filho, M. L. Pereira Filho, IPT - Instituto de Pesquisas Tecnológicas (Brazil)

8785 GD Optical spectroscopy for differentiation of liver tissue under distinct stages of fibrosis: an ex vivo study [8785-77]

D. A. Fabila, L. F. Hernández, J. de la Rosa, S. Stolik, Instituto Politécnico Nacional (Mexico); U. D. Arroyo-Camarena, Hospital General de México (Mexico); M. D. López-Vancell, Univ. Nacional Autónoma de México (Mexico); G. Escobedo, Hospital General de México (Mexico)

Author Index 


\title{
Conference Committees
}

\author{
Conference Chair
}

Manuel Filipe P. C. Martins Costa, Universidade do Minho (Portugal)

Conference Vice-chair

Orlando Frazão, INESC Porto (Portugal)

Technical Chairs:

Gonçalo Figueira, Instituto Superior Técnico (Portugal)

Hélder Crespo, Universidade do Porto (Portugal)

José Figueiredo, Universidade do Algarve (Portugal)

Pedro Jorge, INESC Porto (Portugal)

Rogério Nogueira, Instituto de Telecomunicações, Aveiro (Portugal)

António Baptista, Universidade do Minho (Portugal)

International Advisory Committee:

Anna Consortini, Università degli Studi di Firenze (Italy)

Ángela Guzmán, CREOL, The College of Optics and Photonics, Univ. of

Central Florida (United States)

Carlos Saavedra, Universidad de Concepción (Chile)

Carmiña Londoño, National Science Foundation (United States)

Efraín Solarte Rodriguez, Universidad del Valle (Colombia)

Elizabeth Rogan, The Optical Society

Eric Rosas, Red Iberoamericana de Óptica

Eugene Arthurs, SPIE

Guillermo Baldwin, Pontificia Universidad Católica del Perú (Peru)

Héctor J. Rabal, Centro de Investigaciones Opticas (Argentina)

Herve Lefevre, European Optical Society

Humberto Michinel, Universidad de Vigo (Spain)

José Luis Paz, Universidad Simón Bolívar (Venezuela)

José R. Salcedo, Multiwavephotonics (Portugal)

Juan G. Darias Gonzalez, Centro de Aplicaciones Tecnológicas y

Desarrollo Nuclear (Cuba)

Katrina Svanberg, Lunds Universitet (Sweden)

María Josefa Yzuel Giménez, Universitat Autònoma de Barcelona (Spain)

Maria Luisa Calvo, Universidad Complutense de Madrid (Spain)

Marcelo R. Trivi, Centro de Investigaciones Ópticas (Argentina)

Paulo Fiadeiro, Universidade da Beira Interior (Portugal)

Pedro Andrés, Universitat de València (Spain)

Ramón Rodríguez-Vera, Centro de Investigaciones en Óptica A.C.

(México) 
International Scientific Committee:

Alberto José Fernández Cuervo, Universidad Central de Venezuela (Venezuela)

Alfonso Lastras Martínez, Universidad Autónoma de San Luís Potosí (México)

Amalia Martínez García, Academia Mexicana de Óptica (México) and Centro de Investigaciones en Óptica A.C. (México)

Amparo Pons Martí, Universitat de València (Spain)

Anand Krishna Asundi, Optics and Photonics Society of Singapore (Singapore)

André P. L. Barbero, Universidade Federal Fluminense, Niterói (Brazil)

Andrea Cusano, Università degli Studi del Sannio (Italy)

Andrés Márquez, Universidad de Alicante (Spain)

Andrew Moore, Herriot-Watt University (Scotland)

Angel Augier Calderin, Universidad de La Habana (Cuba)

Armando Dias Tavares, Universidade Federal do Rio de Janeiro (Brazil)

Asticio Vargas, Centro de Óptica y Fotónica de la Universidad de Concepción (Chile)

Clementina Timus, National Institute for Laser, Plasma \& Radiation Physics (Romania)

Carlos Ferreira, Universitat de València (Spain)

Carlos Saavedra, Universidad de Concepción (Chile)

Cesar Costa, Escuela Politécnica Nacional (Ecuador)

Cristiano M. B. Cordeiro, Universidade Estadual de Campinas (Brazil)

Daniel Malacara Hernández, Centro de Investigaciones en Óptica A.C. (México)

Efraín Solarte Rodriguez, RCO, Universidad del Valle (Colombia)

Eric Rosas, Centro Nacional de Metrología de México (México)

Elvira Fortunato, Universidade Nova de Lisboa (Portugal)

Erna Frins, Universidad de la República (Uruguay)

Francisco De Zela, Pontificia Universidad Católica del Perú (Peru)

Fernando Mendoza, Centro de Investigaciones en Óptica A.C. (México)

Gert von Bally, Universität Münster (Germany)

Gilberto Gómez Rosas, Universidad de Guadalajara (México)

Guillermo Baldwin, Pontificia Universidad Católica del Perú (Peru)

Guillermo Kaufmann, Universidad Nacional de Rosario (Argentina)

Ignacio Negueruela, Universidad de Zaragoza (Spain)

Hans Herzig, European Optical Society

Hai-Ning Cui, University of Nanjing (China)

Hector Rabal, Centro de Investigaciones en Ópticas (Argentina)

Humberto Michinel, Universidade de Vigo (Spain)

Jaime Cascante, Universidad de Costa Rica (Costa Rica)

James Wyant, University of Arizona (United States)

Javier Alda, Universidad Complutense de Madrid (Spain)

Jesus Lancis, Universitat Jaume I de Castellón (Spain)

Joanna Schmit, Bruker Nano (United States)

João Manuel Tavares, Universidade do Porto (Portugal) 
Joaquín Campos Acosta, Instituto de Óptica, Consejo Superior de Investigaciones Científicas (Spain) and SEDOPTICA (Spain)

João Crisóstomo Weyl Albuquerque Costa, Universidade Federal do Pará (Brazil)

Jorge Rueda, UniPamplona (Colombia)

Jose Benito Vazquez-Dorrio, Universidade de Vigo (Spain)

José Luis Paz, CTOV (Venezuela)

José Manuel de Nunes Vicente Rebordão, Universidade Nova de Lisboa

(Portugal)

José Ramiro Fernandes, Universidade de Trás-Os-Montes e Alto Douro

(Portugal)

Juan G. Darias Gonzalez, Centro de Aplicaciones Tecnológicas y

Desarrollo Nuclear (Cuba)

Lucilia Cescato, Universidade Estadual de Campinas (Brazil)

Luis Miguel Bernardo, Universidade do Porto (Portugal)

Luis Roso, Centro de Láseres Pulsados Ultracortos Ultraintensos (Spain)

Kiyofumi Matsuda, Advanced Industrial Science and Technology,

Tsukuba (Japan)

Kim Chew Ng, Monash University (Australia)

Manuel Lopez-Amo, Universidad Pública de Navarra (Spain)

Manuel Filipe P. C. Martins Costa, Universidade do Minho (Portugal)

Marcelo Trivi, Universidad Nacional de Mar del Plata (Argentina)

Maria A. G. Martinez, Centro Federal de Educação Tecnológica, Rio de Janeiro (Brazil)

Maria de Jesus Gomes, Universidade do Minho (Portugal)

Maria J. Pontes, Universidade Federal do Espírito Santo, Vitória (Brazil)

Maria Sagrario Millan, Universitat Politècnica de Catalunya (Spain)

Maria T. M. Rocco Giraldi, Instituto Militar de Engenharia, Rio de Janeiro (Brazil)

Mário Vaz, Instituto de Engenharia Mecânica e Gestão Industrial, Porto (Portugal)

Marta Ramos, Universidade do Minho (Portugal)

Maxim Tomilin, University of St. Petersburg (Russian Federation)

Miguel Andrés, Universitat de València (Spain)

Miguel Gonzalez Herraez, Universidad de Alcalá (Spain)

Mikiya Muramatsu, Universidade de São Paulo (Brazil)

Mourad Zghal, La Société Tunisienne d'Optique (Tunisia)

Mustafa Erol, Bozok University (Turkey)

Omar Ormachea, Universidad Privada Boliviana (Bolivia)

Oracio Barbosa García, Centro de Investigaciones en Óptica A.C. (México)

Pablo Artal, Universidad de Murcia (Spain)

Paulo Fiadeiro, Universidade da Beira Interior (Portugal)

Radu Chisleag, Technical University of Bucharest (Romania)

Ramón Rodríguez-Vera, Centro de Investigaciones en Óptica A.C. (México)

Rastogi Pramod, École Polytechnique Fédérale de Lausanne (Switzerland)

Roger Ferlet, Université Paris (France)

Tomas Catunda, Universidade de São Paulo, São Carlos (Brazil) 
William T. Rhodes, Florida Atlantic University (United States)

Yobani Mejía Barbosa, Universidad Nacional de Colombia (Colombia)

Zohra Ben Lakhdar, Université de Tunis El Manar (Tunisia)

Program Committee:

Armando Dias Tavares, Universidade Federal do Rio de Janeiro (Brazil)

Carlos Gerardo Treviño-Palacios Instituto Nacional de Astrofísica, Óptica y Electrónica (México)

Cristiano M. B. Cordeiro, Universidade Estadual de Campinas (Brazil)

Daniel Malacara Hernández, Centro de Investigaciones en Óptica A.C. (México)

Efraín Solarte Rodriguez, RCO, Universidad del Valle (Colombia)

Eric Rosas, Centro Nacional de Metrología de México (México)

Hector Rabal, Centro de Investigaciones en Ópticas (Argentina)

Helder Crespo, Universidade do Porto (Portugal)

Hypolito Kalinowski, Universidade Tecnológica Federal do Paraná (Brazil)

Humberto Michinel, Universidade de Vigo (Spain)

Gonçalo Figueira, Instituto Superior Técnico (Portugal)

Guillermo Baldwin, Pontificia Universidad Católica del Perú (Peru)

Ireneu Dias, INESC Porto (Portugal)

João Manuel Tavares, Universidade do Porto (Portugal)

José Benito Vazquez-Dorrio, Universidade de Vigo (Spain)

José Figueiredo, Universidade do Algarve (Portugal)

José Luis Paz, CTOV (Venezuela)

José Luis Santos, Faculdade de Ciências da Universidade do Porto (Portugal)

José Ramiro Fernandes, Universidade de Trás-Os-Montes e Alto Douro (Portugal)

José Salcedo, Multiwavephotonics (Portugal)

Josué Álvarez Borrego, Centro de Investigación Científica y de

Educación Superior de Ensenada (México)

Lúcia Bilro, Instituto de Telecomunicações, Aveiro (Portugal)

Maité Flores-Arias, Universidade de Santiago de Compostela (Spain)

Manuel Lopez-Amo, Universidad Pública de Navarra (Spain)

Manuel Filipe P. C. Martins Costa, Universidade do Minho (Portugal)

Manuel Joaquim Marques, Faculdade de Ciências da Universidade do

Porto (Portugal)

Marcelo Trivi, Universidad Nacional de Mar del Plata (Argentina)

Maria Sagrario Millan, Universitat Politècnica de Catalunya (Spain)

Marlos Viana, University of Illinois at Chicago (United States)

Marta Ramos, Universidade do Minho (Portugal)

Maxim Tomilin, University of St. Petersburg (Russian Federation)

Miguel Gonzalez Herraez, Universidad de Alcalá (Spain)

Mikhail Vasilevski, Universidade do Minho (Portugal)

Mikiya Muramatsu, Universidade de São Paulo (Brazil)

Mourad Zghal, La Société Tunisienne d'Optique (Tunisia)

Orlando Frazão, INESC Porto (Portugal) 
Paulo Marques, INESC Porto (Portugal) and Faculdade de Ciências da Universidade do Porto (Portugal)

Paulo Tavares, Faculdade de Engenharia da Universidade do Porto (Portugal)

Pedro Jorge, INESC Porto (Portugal)

Pedro Andrés, Universitat de València (Spain)

Pedro Serra, University of Bradford (United Kingdom)

Ramón Rodríguez-Vera, Centro de Investigaciones en Óptica A.C. (México)

Rogério Nogueira, Instituto de Telecomunicações, Aveiro (Portugal) Salvador Bará, Universidade de Santiago de Compostela (Spain)

Vasco Teixeira, Universidade do Minho (Portugal)

Zuqing Zhu, University of Science and Technology of China (China)

Local Organizing Committee:

Ana João Martins (Portugal)

António Baptista (Portugal)

António Lobo (Portugal)

António Teixeira (Portugal)

Armando Tavares (Brazil)

Gonçalo Figueira (Portugal)

Ireneu Dias (Portugal)

João Pinto Coelho (Portugal)

Jesús Blanco (Spain)

Joaquim Carneiro (Portugal)

Jose Benito Vazquez-Dorrio (Spain)

José Figueiredo (Portugal)

José Luis Santos (Portugal)

José Manuel Baptista (Portugal)

José Ramiro Fernandes (Portugal)

José R. Salcedo (Portugal)

Lucia Bilro (Portugal)

Luis Marques (Portugal)

Manuel Filipe Costa (Portugal)

Manuel Jorge Marques (Portugal)

Manuel Joaquim Marques (Portugal)

Mário Lima (Portugal)

Mário Vaz (Portugal)

Marta Miranda (Spain)

Mikiya Muramatsu (Brazil)

Orlando Frazão (Portugal)

Paulo Marques (Portugal)

Paulo Roriz (Portugal)

Pedro Jorge (Portugal)

Pedro Serra (United Kingdom)

Paulo Tavares (Portugal)

Raquel Queirós (Portugal)

Rogério Nogueira (Portugal) 
Rosa Romero (Portugal)

Susana Silva (Portugal))

xxxvi

Proc. of SPIE Vol. $8785878501-35$

Downloaded From: https://www.spiedigitallibrary.org/conference-proceedings-of-spie on 26 Apr 2023 Terms of Use: https://www.spiedigitallibrary.org/terms-of-use 


\section{Introduction}

The VIII Reunión Iberoamericana de Óptica / XI Reunión Iberoamericana de Óptica, Láseres y Aplicaciones - VIII Reunião Iberoamericana de Óptica/XI Reunião Iberoamericana de Óptica, Lasers e Aplicações, RIAO/OPTILAS 2013, ran very successfully in an exciting friendly and most pleasant way. Four hundred forty one (441) participants from 39 countries openly shared ideas and experiences discussing the remarkable and exciting developments in the last the three years in Optics in Iberoamerica presented at the conference.

The conference occurred July 22 to 26,2013 at the University of Porto in Portugal. Twenty one years after the first Iberoamerican Optics Meeting (Reunión Iberoamericana de Óptica - Reunião Iberoamericana de Óptica, RIAO) in Barcelona, the reference meeting of the Iberoamerican Optics community returned to the Iberian Peninsula. It was the greatest of the honors for us, the Portuguese Society for Optics and Photonics (SPOF), to welcome colleagues from all over lberoamerica and the world to Portugal renewing friendships and growing many more!

The success of the conference was only possible with the active support and commitment of the Red(e) Iberoamericana de Optica, the Spanish Optical Society, SEDOPTICA, the Mexican Academy of Optics, AMO, the Colombian Optics Network, RCO, the Venezuelan Optics Committee, CVO, and many colleagues and institutions from all over Iberoamerica. The endorsement and active support of the most important international scientific optics societies: ICO, SPIE, and OSA, represented at the highest level, and of several major institutions: Photonics21, EOS, LAM, SECPhO, EPIC, and national societies, committees and boards: STO, OPSS, APLO, SPF, FCT, as well as the SPIE and OSA student chapters of the University of Porto and the University of Aveiro, INESC, and the University of Porto was fundamental and much appreciated.

From the 605 works accepted, 581 were effectively presented. 13 high level plenary lectures, 61 key lectures covering all fields of optics and photonics, 135 oral communications and 372 posters were presented and attended by 441 participants, including over 158 students, from 39 countries all over Iberoamerica and the world. Two hundred fifty one (251) full papers were accepted for publication in the proceedings book. Two very interesting special sessions and round tables on "Entrepreneurship in Optics \& Photonics" and "Women \& Optics" were organized with strong active participation of the audience. These sessions were jointly organized between RIAO/OPTILAS 2013 and ETOP2013. The 12 Education and Training in Optics and Photonics Conference was very successfully organized in parallel and in an articulated way with RIAO/OPTILAS 2013. The participation of students (158) and young researchers was very positive and a very good sign of the growth potential of our Iberoamerican O\&P research. SPIE 
and OSA student prizes were awarded. RIAO/OPTILAS2013 also held the ceremony of the IUPAP Prize 2012 awarded to Nirit Dudovich, Weizmann Institute, Israel for the work "Resolving and manipulating attosecond processes via strongfield light-matter interactions".

The board of the Red(e) Iberoamericana de Optica met during the conference and Prof. Dr. Pedro Andrés Bou was sworn in as the new president. The recent activities of the Red(e) and very positive prospects of evolution were presented during the general assembly of RIAO/OPTILAS.

RIAO/OPTILAS, the reference meeting in optics in Iberoamerica and our main "window" to the world, will keep growing and re-enforce itself. The next edition to be held in Chile in the fall of 2016 will be another great success reflective of the decisive importance of Iberoamerica's contribution to the development of optics and photonics.

Hope to see you all there!

Manuel Filipe P. C. Martins Costa 